\title{
Time-Reversal Detection Using Antenna Arrays
}

\author{
Yuanwei Jin, Senior Member, IEEE, and José M. F. Moura, Fellow, IEEE
}

\begin{abstract}
The paper studies detection of a target buried in a rich scattering medium by time reversal. We use a multi-static configuration with receive and transmit arrays of antennas. In time reversal, the backscattered field is recorded, time reversed, and retransmitted (mathematically or physically) into the same scattering medium. We derive two array detectors: the time-reversal channel matched filter when the target channel response is known; and the time-reversal generalized-likelihood ratio test (TR-GLRT) when the target channel response is unknown. The noise added in the initial probing step to the time-reversal signal makes the analysis of the TR-GLRT detector non trivial. The paper derives closed form expressions for the signal-to-noise ratio gain provided by this detector over the corresponding conventional clutter subtraction energy detector in the two extreme conditions of weak and strong (electronic additive) noise and shows that time reversal provides, under weak noise, the optimal waveform shape to probe the environment. We analyze the impact of the array configuration on the detection performance. Finally, experiments with electromagnetic data collected in a multipath scattering laboratory environment confirm our analytical results. Under the realistic conditions tested, time reversal provides detection gains over conventional detection that range from 2 to $4.7 \mathrm{~dB}$.
\end{abstract}

Index Terms-Adaptive waveforms, antenna array, detection, imaging, localization, radar, sonar, synthetic aperture radar (SAR), time reversal.

\section{INTRODUCTION}

$\mathbf{I}$ $\mathrm{N}$ time reversal (TR), a short pulse transmitted by a source through a dispersive medium is received by an array, then time reversed, energy normalized, and retransmitted (mathematically or physically) through the same channel. If the scattering channel is reciprocal and rich in multipath, the retransmitted signal refocuses on the original source. A rich scattering channel is highly dispersive and exhibits significant multipath propagation. How rich the channel is can be assessed by the channel type [1], [2], i.e., the empirical distribution of the (magnitude) of the channel response. TR has drawn considerable attention in ultrasound and acoustics [2]-[13], electromagnetics [14], [15], and in algorithm development for TR based methods, e.g., [16]-[18]. In [2], we studied TR based detection for a single

Manuscript received February 02, 2008; revised November 14, 2008. First published December 09, 2008; current version published March 11, 2009. The associate editor coordinating the review of this manuscript and approving it for publication was Prof. Olivier Besson. This work is funded by the Defense Advanced Research Projects Agency through the Army Research Office under Grant W911NF-04-1-0031.

Y. Jin is with Department of Engineering and Aviation Sciences, University of Maryland Eastern Shore, Princess Anne, MD 21853 USA (e-mail: yjin@umes. edu).

J. M. F. Moura is with Department of Electrical and Computer Engineering, Carnegie Mellon University, Pittsburgh, PA 15213 USA (e-mail: moura@ece. cmu.edu).

Color versions of one or more of the figures in this paper are available online at http://ieeexplore.ieee.org.

Digital Object Identifier 10.1109/TSP.2008.2010425 antenna pair and evaluated its performance analytically and experimentally. This paper extends the single antenna TR detection to the general framework where arrays of antennas are utilized; preliminary versions of parts of this work are in [12] and [13].

Before we begin, we clarify the terms involving clutter and rich scattering environment in this paper. In this paper, clutter refers to unwanted scatterers within a scene of interest. We assume that the clutter can be reliably estimated and subtracted out from the measurement. The presence of a large amount of unwanted scatterers creates a highly dispersive background Green's function for target detection. This background Green's function characterizes the highly scattering environment. This paper studies the problem of detecting a target by time reversal in rich scattering.

We consider two antenna arrays $A$ and $B$ that form a multistatic configuration. Both can operate in transmit and receive modes. TR detection involves three steps:

1) First, we probe the environment when no target is present. A wideband signal is transmitted from each of the transmitting antennas of array $A$. The received signals are recorded by array $B$. This step, repeated $M$ times to average the noise, learns the clutter response matrix.

2) We then monitor the scene to detect if a target or targets entered the space of interest. When targets are present, the measurements correspond to the target plus clutter response matrix. The clutter response matrix learned in step 1 can be subtracted out from the measurements. If a target is present, the residue signals contain the effective target response at the array $B$.

3) Finally, the residue signals received at array $B$ are timereversed, energy normalized, retransmitted simultaneously from all antennas at $B$ back into the same medium, and recorded at the antennas at array $A$. The component of the signal solely due to clutter is again subtracted out, which results in the residue target signals at array $A$. The test statistics based on these residue signals are calculated and compared with a predetermined threshold. A decision is made whether a target is present or not.

We study two different scenarios where the target channel response is 1) known or 2) unknown. For both scenarios, we first derive the detectors and then study their performance. For scenario 1, we present the TR detector that is matched to the known target channel response. For scenario 2, due to the noise that may contaminate the measurements, we estimate the target channel response first and then derive the TR generalized-likelihood ratio test (TR-GLRT). We provide an approximate closed form of the maximum-likelihood estimate of the target channel response.

To benchmark the gains provided by TR detectors over conventional detectors, we quantify the signal-to-noise ratio (SNR) 
detection gain when detecting a target. For the TR-GLRT, this analysis is not tractable in general. Instead, we develop closed forms for the maximal SNR gain $\left(\mathrm{SNRG}_{\max }\right)$ derived when the noise is weak, and for the asymptotic SNR gain $\left(\mathrm{SNRG}_{\text {asym }}\right)$, asymptotic in the sense of large number of samples, when the noise is strong. $\mathrm{SNRG}_{\max }$ quantifies the performance advantage of TR detection over conventional detection when the target channel response is known; $\mathrm{SNRG}_{\text {asym }}$ characterizes the performance advantage of TR detection over conventional detection when the target channel is unknown and is estimated from a large number of data samples. Unlike in the single antenna pair detection problem, the employment of antenna arrays provides additional degrees of freedom and allows trading off frequency bandwidth against different array configurations. The analysis using $\mathrm{SNRG}_{\max }$ indicates that the time-reversal detector has a significant gain over the conventional detector. We explain this gain by showing that TR based detection performs optimal matching at the transmitter, i.e., it adaptively matches the transmitted waveform to the target channel. The analysis using $\mathrm{SNRG}_{\text {asym }}$ provides the gain afforded by the TR-GLRT over the conventional detector when the unknown channel is estimated with an asymptotic large number of samples and the impact that noise and array configuration have on time-reversal detection. For the same level of scattering, the detection gain afforded by time reversal over conventional detection reduces as the array gets larger.

Our analytical results are validated with electromagnetic data collected in a cluttered laboratory. These experiments confirm that, for the realistic conditions tested, TR array detection provides significant gain over conventional array detection, ranging from 2 to $4.7 \mathrm{~dB}$. To further explore TR, we present detection results with a bistatic synthetic aperture radar (SAR) configuration with and without TR (TR-SAR). We show that TR-SAR provides a gain over conventional SAR of about $2 \mathrm{~dB}$ using a baseline of ten antennas. We also show how this gain is impacted by the noise level in the time reversed signal, i.e., how noisy the time reversed step is. The additive noise in the time reversed signal reduces the channel estimation accuracy, reducing the detection gain of TR-SAR over conventional SAR detection.

The paper is organized as follows. Section II establishes the TR signal model. Section III formulates both the TR and the conventional detection problems. We then present the corresponding test statistics. Section IV derives closed forms for the false alarm rate and detection probabilities. Section V shows the connection between TR and optimal waveform shaping. Section VI discusses the maximal SNR gain (weak noise) and the asymptotic SNR gain (strong noise) of TR detection over conventional detection. Section VII presents experimental results, including TR-SAR versus conventional SAR. We summarize our conclusions in Section VIII.

Notation: Lower and upper case boldface letters denote vectors and matrices, respectively; $(\cdot)^{*},(\cdot)^{T}$, and $(\cdot)^{H}$ stand for conjugate, transpose, and Hermitian transpose, respectively; $\mathfrak{R}(\mathbf{A})$ and $\mathfrak{I}(\mathbf{A})$ are the real and the imaginary parts of $\mathbf{A}$; $\operatorname{vec}\{\mathbf{A}\}$ is the column vector obtained by stacking the columns of the matrix $\mathbf{A} ; \mathbf{I}_{m}$ is the identity matrix of order $m ; \mathbf{e}_{p}$ is a column vector of zero entries except that its $p$ th element is 1 ; $\operatorname{diag}\{\mathbf{x}\}$ is the diagonal matrix whose diagonal collects the en- tries of the vector $\mathbf{x} ; \operatorname{Tr}\{\cdot\}$ and $\operatorname{det}(\mathbf{A})$ are the trace and determinant of matrix $\mathbf{A} ;\|\cdot\|$ is the vector or matrix Frobenius norm; $\mathrm{E}\{\cdot\}$ is the expected value of a random quantity; and $\odot$ is the Hadamard product of two vectors or matrices, i.e., the vector or matrix of their components wise product.

\section{TR DATA MODEL}

We extend the single-antenna-pair TR detection results in [2] to time-reversal detection with arrays of antennas. We consider an active radar (or sonar) system with a pair of antenna arrays $A$ and $B$ in a multi-static configuration. Array $A$ has $P$ antennas $A_{1}, \ldots, A_{P}$, and array $B$ has $N$ antennas $B_{1}, \ldots, B_{N}$. The arrays $A$ and $B$ can switch between transmit mode and receive mode. Suppose that the initial transmission starts from the array $A$. The signals transmitted from antennas $A_{p}, p=1, \ldots, P$ are $s_{p}(t)$. For simplicity, we will often assume that $s_{p}(t)=s(t)$. The transmitted signal $s_{p}(t)$ is wideband, with duration $2 T$, and bandwidth $\mathrm{BW}=(2 \pi) /(T)$. This signal $s_{p}(t)$ is considered wideband if the signal bandwidth, BW, is much larger than the coherence bandwidth, $B_{c}$, of the scattering channel. The coherence bandwidth is given approximately by the inverse of the largest channel delay spread [19]. The discrete Fourier representation of the signal $s_{p}(t)$ is

$$
S_{p}\left(\omega_{q}\right)=\sum_{n=1}^{Q-1} x_{p, n} e^{-j \frac{2 \pi}{Q} q n}
$$

where $x_{p, n}$ is the $n$th discrete time domain sample

$$
\omega_{q}=\frac{2 \pi}{T(Q-1)}\left(q_{1}+q\right), \quad q=0, \ldots, Q-1
$$

and $q_{1}$ is a constant. For real valued time domain signals, the causal representation of its time-reversal is $s_{p}\left(T_{c}-t\right)$, where $T_{c}$ is the chosen time window length. Note that $T_{c}$ is dependent on the channel dispersiveness. For a rich multipath channel, $T_{c} \gg$ $T$. Thus, the discrete Fourier representation of $s_{p}\left(T_{c}-t\right)$ is

$$
e^{j \omega_{q} T_{c}} S_{p}^{*}\left(\omega_{q}\right) \forall q .
$$

In what follows, we use the discrete frequency representation to describe the time-reversal signal model and omit the linear phase term $e^{j \omega_{q} T_{c}}$.

In typical radar stationary target configurations, the propagation and scattering of electromagnetic waves are modeled as linear processes. We introduce the following two channel frequency response matrices. The $N \times P$ clutter channel frequency response matrix

$$
\mathbf{H}_{c}\left(\omega_{q}\right)=\left[\mathbf{H}_{c, n p}\left(\omega_{q}\right)\right], \quad q=0, \ldots, Q-1
$$

is the response of the channel when no target is present. Its $(n, p)$ th entry is the channel response from antenna $A_{p}$ to antenna $B_{n}$ at angular frequency $\omega_{q}=2 \pi f_{q}$. We assume that the radar channel is reciprocal. Then $\mathbf{H}_{c, n p}$ also represents the channel response from antenna $B_{n}$ to antenna $A_{p}$. Similarly, the $N \times P$ target + clutter channel frequency response $\mathbf{H}_{t+c}\left(\omega_{q}\right)$, $q=0, \ldots, Q-1$, is the channel response when one or more targets and clutter are both present. Let

$$
\mathbf{H}\left(\omega_{q}\right)=\mathbf{H}_{t+c}\left(\omega_{q}\right)-\mathbf{H}_{c}\left(\omega_{q}\right) .
$$


This denotes the difference between the channel response when a single or multiple targets are present and the channel response when no target is present; it represents the effective target response, including the secondary scattering due to the interaction between the surrounding scatterers and the target. Throughout the paper, it is assumed that the clutter channel frequency response $\mathbf{H}_{c}\left(\omega_{q}\right), \forall q$, is learned and subtracted out from the measurements (see a similar treatment in [2]). It suffices to focus on the target response $\mathbf{H}\left(\omega_{q}\right), \forall q$, to study the detection problem.

\section{A. Data Organization}

We will benchmark TR detection with respect to a conventional detection problem. This section organizes the data for both problems. We consider first the transmission from the $P$ antennas at array A received by the $N$ antennas at array B. We will aggregate the data received at the $N$ antennas in $\mathrm{B}$, say $Z_{m, n}\left(\omega_{q}\right)$, in $N$-dimensional vectors $\mathbf{z}_{m}\left(\omega_{q}\right)$, whose components sum up the $P$ received signals $Z_{m, p, n}\left(\omega_{q}\right)$ at frequency $\omega_{q}, 0 \leq q \leq Q-1$, transmitted $m$ th snapshot, $1 \leq m \leq M$, by the $p$ th antenna of A. Each of the vectors $\mathbf{z}_{m}\left(\omega_{q}\right)$ is indexed by $m$ and is a function of $\omega_{q}$. We then stack these vectors for the $Q$ frequencies to obtain an $N Q$-dimensional vector, say $\mathbf{z}_{m}$. Finally, we collect a total of $M$ vectors to form the $N Q \times M$ dimensional Gram data matrix, say $\mathbf{Z}$. We now explain this in detail.

1) TR Data: In TR detection, there are two steps: the direct step and the TR step. We assume that in either step, there are $M$ snapshots. We discuss first the direct step.

Direct Step: For the $m$ th snapshot, $m=1, \ldots, M$, let $Y_{m, p, n}\left(\omega_{q}\right)$ be the signal received at antenna $B_{n}$ transmitted from antenna $A_{p}$ at frequency $\omega_{q}$. Stacking these measurements at all the $N$ antennas of array $B$ in the $N$-dimensional vector

$$
\begin{aligned}
\forall m, p: \mathbf{y}_{m, p}\left(\omega_{q}\right) & =\left[Y_{m, p, 1}\left(\omega_{q}\right), \ldots, Y_{m, p, N}\left(\omega_{q}\right)\right]^{T} \\
& =\mathbf{H}\left(\omega_{q}\right) \mathbf{e}_{p} S_{p}\left(\omega_{q}\right)+\mathbf{v}_{m, p}\left(\omega_{q}\right)
\end{aligned}
$$

Equation (3) expresses these measurements in terms of the $p$ th-column of the target channel response $\mathbf{H}\left(\omega_{q}\right)$; the scalar Fourier representation $S_{p}\left(\omega_{q}\right)$ of the signal is given in (1); and the noise vector at frequency $\omega_{q}$ is $\mathbf{v}_{m, p}\left(\omega_{q}\right)$. The signal $\mathbf{y}_{m}\left(\omega_{q}\right)$ received at array $B$ is the superposition of all the signals transmitted from the $P$ antennas at array $A$; it is

$$
\begin{aligned}
\mathbf{y}_{m}\left(\omega_{q}\right) & =\sum_{p=1}^{P} \mathbf{y}_{m, p}\left(\omega_{q}\right), \\
& =\mathbf{H}\left(\omega_{q}\right) \mathbf{s}_{A}\left(\omega_{q}\right)+\mathbf{v}_{m}\left(\omega_{q}\right) \\
\mathbf{s}_{A}\left(\omega_{q}\right) & =\left[S_{1}\left(\omega_{q}\right), \ldots, S_{P}\left(\omega_{q}\right)\right]^{T} \\
\mathbf{v}_{m}\left(\omega_{q}\right) & =\left[V_{m, 1}\left(\omega_{q}\right), \ldots, V_{m, N}\left(\omega_{q}\right)\right]^{T}
\end{aligned}
$$

where $\mathbf{s}_{A}\left(\omega_{q}\right)$ and $\mathbf{v}_{m}\left(\omega_{q}\right)$ are the signal and noise vector components of $\mathbf{y}_{m}\left(\omega_{q}\right)$, respectively; and $V_{m, n}\left(\omega_{q}\right)$ is the additive noise in the $m$ th data snapshot at antenna $n$, frequency $\omega_{q}$. Collecting for each snapshot $m$, the frequency response $\mathbf{y}_{m}\left(\omega_{q}\right)$ in an $N Q$-dimension vector $\mathbf{y}_{m}$ yields

$$
\begin{aligned}
\mathbf{y}_{m} & =\left[\mathbf{y}_{m}^{T}\left(\omega_{0}\right), \ldots, \mathbf{y}_{m}^{T}\left(\omega_{Q-1}\right)\right]^{T} \\
& =\mathbf{H s}_{A}+\mathbf{v}_{m}
\end{aligned}
$$

$$
\begin{aligned}
\mathbf{H} & =\operatorname{diag}\left[\mathbf{H}\left(\omega_{0}\right), \ldots, \mathbf{H}\left(\omega_{Q-1}\right)\right]_{N Q \times P Q} \\
\mathbf{s}_{A} & =\left[\mathbf{s}_{A}^{T}\left(\omega_{0}\right), \ldots, \mathbf{s}_{A}^{T}\left(\omega_{Q-1}\right)\right]^{T} \\
\mathbf{v}_{m} & =\left[\mathbf{v}_{m}^{T}\left(\omega_{0}\right), \ldots, \mathbf{v}_{m}^{T}\left(\omega_{Q-1}\right)\right]^{T} .
\end{aligned}
$$

The block diagonal matrix $\mathbf{H}$ is $N Q \times P Q$, and the signal $\mathbf{s}_{A}$ and noise $\mathbf{v}_{m}$ vectors are $P Q$ - and $N Q$-dimensional, respectively. We define the signal energy $E_{s}$ and the total energy $E_{T}$ transmitted by array $A$

$$
\begin{aligned}
& E_{s}=\frac{1}{Q} \sum_{q=0}^{Q-1}\left|S_{p}\left(\omega_{q}\right)\right|^{2} \\
& E_{T}=\frac{1}{Q}\left\|\mathbf{s}_{A}\right\|^{2}=P E_{s}
\end{aligned}
$$

where we assume that all antennas $A_{p}$ transmit the same energy. We now discuss the TR step.

TR Step: In the TR step, each data vector $\mathbf{y}_{m}$ is time reversed, energy normalized, and retransmitted back from array $B$ to array $A$. The $P Q$-dimensional signal vector at array $A$ is given by

$$
\begin{aligned}
\mathbf{x}_{m} & =k_{m} \mathbf{H}^{T} \mathbf{y}_{m}^{*}+\mathbf{w}_{m} \\
& =k_{m} \mathbf{H}^{T} \mathbf{H}^{*} \mathbf{s}_{A}^{*}+k_{m} \mathbf{H}^{T} \mathbf{v}_{m}^{*}+\mathbf{w}_{m}
\end{aligned}
$$

where the received signal and noise vectors are

$$
\begin{aligned}
\mathbf{x}_{m} & =\left[\mathbf{x}_{m}^{T}\left(\omega_{0}\right), \ldots, \mathbf{x}_{m}^{T}\left(\omega_{Q-1}\right)\right]^{T} \\
\mathbf{x}_{m}\left(\omega_{q}\right) & =\left[X_{m, 1}\left(\omega_{q}\right), \ldots, X_{m, P}\left(\omega_{q}\right)\right]^{T} \\
\mathbf{w}_{m} & =\left[\mathbf{w}_{m}^{T}\left(\omega_{0}\right), \ldots, \mathbf{w}_{m}^{T}\left(\omega_{Q-1}\right)\right]^{T} \\
\mathbf{w}_{m}\left(\omega_{q}\right) & =\left[W_{m, 1}\left(\omega_{q}\right), \ldots, W_{m, P}\left(\omega_{q}\right)\right]^{T} .
\end{aligned}
$$

In (13), due to reciprocity, the target channel response $\mathbf{H}^{T}$ between array $B$ and array $A$ is the transpose of the target channel response between arrays $A$ and $B$. The scalar $k_{m}$ is the energy normalization factor at each antenna $B_{n}$

$$
k_{m}=\sqrt{\frac{\left\|\mathbf{s}_{A}\right\|^{2}}{\left\|\mathbf{y}_{m}\right\|^{2}}} .
$$

For the TR detection problem, we concatenate $\mathbf{y}_{m}^{*}$ and $\mathbf{x}_{m}$ in the $Q(P+N) \times 1$-vector

$$
\mathbf{z}_{m}=\left[\begin{array}{ll}
\left(\mathbf{y}_{m}^{*}\right)^{T} & \mathbf{x}_{m}^{T}
\end{array}\right]^{T}
$$

where we used $\mathbf{y}_{m}^{*}$ rather than $\mathbf{y}_{m}$. Finally, the Gram data matrix in TR is described by

$$
\mathbf{Z}=\left[\mathbf{z}_{1}, \ldots, \mathbf{z}_{M}\right] .
$$

2) Conventional Detection Data: When we consider conventional detection, we probe the target channel using identical signals $\mathbf{s}_{A}$ from the array $A$ and $\mathbf{s}_{B}$ from the array $\mathrm{B}$, respectively. Thus, the conventional detection is formulated based upon the measurement pair $\left(\mathbf{y}_{m}, \mathbf{r}_{m}\right)$, where $\mathbf{y}_{m}$ is defined in (7), and $\mathbf{r}_{m}$ is given by

$$
\mathbf{r}_{m}=\beta \mathbf{H}^{T} \mathbf{s}_{B}+\mathbf{w}_{m}
$$

where

$$
\begin{aligned}
\mathbf{r}_{m} & =\left[\mathbf{r}_{m}^{T}\left(\omega_{0}\right), \ldots, \mathbf{r}_{m}^{T}\left(\omega_{Q-1}\right)\right]^{T} \\
\mathbf{r}_{m}\left(\omega_{q}\right) & =\beta \mathbf{H}^{T}\left(\omega_{q}\right) \mathbf{s}_{B}\left(\omega_{q}\right)+\mathbf{w}_{m}\left(\omega_{q}\right)
\end{aligned}
$$


TABLE I

LIST OF FOUR DETECTORS

\begin{tabular}{lll}
\hline & TR Detection & Conventional Detection \\
\hline Ideal Scenario & Channel Matched Filter (TRCMF) & Channel Matched Filter (CDCMF) \\
Realistic Scenario & GLRT (TR-GLRT) & GLRT (CD-GLRT) \\
\hline
\end{tabular}

$$
\begin{aligned}
\mathbf{s}_{B} & =\left[\mathbf{s}_{B}^{T}\left(\omega_{0}\right), \ldots, \mathbf{s}_{B}^{T}\left(\omega_{Q-1}\right)\right]^{T} \\
\mathbf{s}_{B}\left(\omega_{q}\right) & =\left[S_{1}\left(\omega_{q}\right), \ldots, S_{N}\left(\omega_{q}\right)\right]^{T} .
\end{aligned}
$$

The subscript $B$ denotes the illuminating signal transmitted from array $B$. The energy normalization factor $\beta$ is

$$
\beta=\sqrt{\frac{\left\|\mathbf{s}_{A}\right\|^{2}}{\left\|\mathbf{s}_{B}\right\|^{2}}}=\sqrt{\frac{P}{N}} .
$$

Like for TR, we concatenate the data $\mathbf{y}_{m}$ and $\mathbf{r}_{m}$ into a vector $\mathbf{u}_{m}$ and collect all the available data in the Gram data matrix $\mathbf{U}$, defined by

$$
\begin{aligned}
\mathbf{u}_{m} & =\left[\begin{array}{ll}
\mathbf{y}_{m}^{T} & \mathbf{r}_{m}^{T}
\end{array}\right]^{T} \\
\mathbf{U} & =\left[\mathbf{u}_{1}, \ldots, \mathbf{u}_{M}\right] .
\end{aligned}
$$

\section{B. Data Statistics}

We collect the data statistics for both the TR and conventional detection problems. The $N Q$-dimensional noise vectors $\mathbf{v}_{m}$ and $\mathbf{w}_{m}$ are circular complex Gaussian random vectors across $Q$-frequencies and $N$-array elements. Their probability densities are

$$
\begin{aligned}
\mathbf{v}_{m} & \sim \mathcal{C N}\left(0, \sigma_{v}^{2} \mathbf{I}_{N Q}\right) \\
\mathbf{w}_{m} & \sim \mathcal{C N}\left(0, \sigma_{w}^{2} \mathbf{I}_{Q P}\right) .
\end{aligned}
$$

The real and imaginary components,[20], of $\mathbf{v}_{m}$ are

$$
\begin{aligned}
\mathfrak{R}\left\{\mathbf{v}_{m}\right\} & \sim \mathcal{N}\left(0, \frac{\sigma_{v}^{2}}{2} \mathbf{I}_{N Q}\right) \\
\mathfrak{I}\left\{\mathbf{v}_{m}\right\} & \sim \mathcal{N}\left(0, \frac{\sigma_{v}^{2}}{2} \mathbf{I}_{N Q}\right) .
\end{aligned}
$$

Similar expressions hold for the real and imaginary components of $\mathbf{w}_{m}$, with $\sigma_{v}$ replaced by $\sigma_{w}$. The two noises $\mathbf{v}_{m}$ and $\mathbf{w}_{m}$ are statistically independent and independent of the transmitted signal. From the assumptions on the noise, the statistics of the data $\mathbf{y}_{m}, \mathbf{r}_{m}$, and $\mathbf{x}_{m}$ follow:

$$
\begin{aligned}
\mathbf{y}_{m} & \sim \mathcal{C N}\left(\mathbf{H s}_{A}, \sigma_{v}^{2} \mathbf{I}_{N Q}\right) \\
\mathbf{r}_{m} & \sim \mathcal{C N}\left(\beta \mathbf{H}^{T} \mathbf{s}_{B}, \sigma_{w}^{2} \mathbf{I}_{Q P}\right) \\
\mathbf{x}_{m} & \sim \mathcal{C N}\left(k_{m} \mathbf{H}^{T} \mathbf{H}^{*} \mathbf{s}_{A}, \sigma_{v}^{2} k_{m}^{2} \mathbf{H}^{T} \mathbf{H}^{*}+\sigma_{w}^{2} \mathbf{I}_{Q P}\right)
\end{aligned}
$$

where the statistics of $\mathbf{x}_{m}$ are conditioned on the gain $k_{m}$. The statistics of $\mathbf{z}_{m}$ are given in Appendix I.

\section{DETECTORS}

We pose the binary hypothesis test where under the null hypothesis $\mathbb{H}_{0}$ the data are target signal free, while under the alternative hypothesis $\mathbb{H}_{1}$ the data contain a target signal. We consider two detection problems: TR detection that uses the data matrix $\mathbf{Z}$ and conventional detection that uses the data matrix U. For the two detection problems, the transmitted signals are energy normalized so that, for benchmarking purposes, the average signal transmission energy is the same in both detection problems. Within each of these detection problems, we consider two scenarios: i) the ideal scenario where the target channel response $\mathbf{H}\left(\omega_{q}\right)$ is assumed known and ii) the realistic scenario where the target channel response $\mathbf{H}\left(\omega_{q}\right)$ is unknown and is estimated from the data. Similar to the discussion in [2], we study the four Neyman-Pearson detectors [21], listed in Table I.

\section{A. TR Channel Matched Filter (TRCMF)}

This simplistic scenario enables deriving the detection gain of TR over conventional detection. We assume that the noise $\mathbf{v}_{m}=\mathbf{0}$ and the target channel response $\mathbf{H}\left(\omega_{q}\right)$ is known. In fact, from (4), if the noise $\mathbf{v}_{m}=\mathbf{0}, \mathbf{H}\left(\omega_{q}\right)$ can be estimated perfectly. Under these assumptions, only the data vector $\mathbf{x}_{m}\left(\omega_{q}\right)$ is relevant. We normalize the energy by

$$
k_{m} \equiv k=\sqrt{\frac{Q P E_{s}}{\left\|\mathbf{H} \mathbf{s}_{A}\right\|^{2}}} .
$$

The binary hypothesis test is

$$
\begin{aligned}
& \mathbb{H}_{1}: \mathbf{x}_{m}=k_{m} \mathbf{H}^{T} \mathbf{H}^{*} \mathbf{s}_{A}^{*}+\mathbf{w}_{m} \\
& \mathbb{H}_{0}: \mathbf{x}_{m}=\mathbf{w}_{m} .
\end{aligned}
$$

The likelihood ratio test yields [22]

$$
\ell_{\mathrm{TRCMF}}(\mathbf{X})=\mathfrak{R}\left(\frac{\left(\mathbf{H}^{T} \mathbf{H}^{*} \mathbf{s}_{A}^{*}\right)^{H} \sum_{m=1}^{M} \mathbf{x}_{m}}{\sigma_{w}\left\|\mathbf{H}^{T} \mathbf{H}^{*} \mathbf{s}_{A}^{*}\right\|}\right)
$$

This detector is a channel matched filter, i.e., it is matched to the known signal component $\mathbf{H}^{T} \mathbf{H}^{*} \mathbf{s}_{A}^{*}$ at the output of the channel.

\section{B. Conventional Detection Channel Matched Filter (CDCMF)}

This is the ideal conventional detection problem given by

$$
\begin{aligned}
& \mathbb{H}_{1}: \mathbf{r}_{m}=\beta \mathbf{H}^{T} \mathbf{s}_{B}+\mathbf{w}_{m} \\
& \mathbb{H}_{0}: \mathbf{r}_{m}=\mathbf{w}_{m} .
\end{aligned}
$$

The target channel response $\mathbf{H}$ is assumed to be known. The likelihood ratio test statistic, [22], for the CDCMF detector is

$$
\ell_{\mathrm{CDCMF}}(\mathbf{R})=\mathfrak{R}\left(\frac{\left(\beta \mathbf{H}^{T} \mathbf{s}_{B}\right)^{H} \sum_{m=1}^{M} \mathbf{r}_{m}}{\sigma_{w}\left\|\beta \mathbf{H}^{T} \mathbf{s}_{B}\right\|}\right)
$$

which, again, is matched to the known signal component $\mathbf{H}^{T} \mathbf{s}_{B}$.

\section{TR Generalized Likelihood Ratio Test (TR-GLRT)}

The detection problem is

$$
\begin{aligned}
\mathbb{H}_{1}: \mathbf{z}_{m} & =\left[\begin{array}{c}
\mathbf{H}^{*} \mathbf{s}_{A}^{*} \\
k_{m} \mathbf{H}^{T} \mathbf{H}^{*} \mathbf{s}_{A}
\end{array}\right]+\left[\begin{array}{c}
\mathbf{v}_{m}^{*} \\
k_{m} \mathbf{H}^{T} \mathbf{v}_{m}^{*}+\mathbf{w}_{m}
\end{array}\right] \\
\mathbb{H}_{0}: \mathbf{z}_{m} & =\left[\begin{array}{c}
\mathbf{v}_{m}^{*} \\
\mathbf{w}_{m}
\end{array}\right] .
\end{aligned}
$$


In (40), the channel response $\mathbf{H}$, is assumed to be unknown. We develop the generalized likelihood ratio test where we first estimate the channel response $\widehat{\mathbf{H}}$. This is difficult because $\mathbf{H}$, besides appearing explicitly, also affects the energy normalization gain $k_{m}$. We approximate the problem by taking the power normalization factor $k_{m}$ to be deterministic. The validation of this assumption is in Appendix III. The impact of the noise that is in the time-reversed signal is studied in Section VI-B.

The TR generalized likelihood ratio test (TR-GLRT) statistic, derived in Appendix I, is

$$
\begin{array}{r}
\ell_{\mathrm{TR}-\mathrm{GLRT}}(\mathbf{Z}) \\
=\frac{1}{\sigma_{v}^{2} \sigma_{w}^{2}} \sum_{m=1}^{M}\left\{2 \Re\left\{k_{m} \sigma_{v}^{2} \mathbf{y}_{m}^{T} \widehat{\mathbf{H}}^{*} \mathbf{x}_{m}+\sigma_{w}^{2} \mathbf{y}_{m}^{T} \widehat{\mathbf{H}}^{*} \mathbf{s}_{A}^{*}\right\}\right. \\
\left.-\sigma_{w}^{2}\left\|\widehat{\mathbf{H}}^{*} \mathbf{s}_{A}^{*}\right\|^{2}-k_{m}^{2} \sigma_{v}^{2}\left\|\widehat{\mathbf{H}}^{T} \mathbf{y}_{m}^{*}\right\|^{2}\right\}
\end{array}
$$

where $\widehat{\mathbf{H}}$ is the channel estimate [see (141) in Appendix I].

\section{Conventional Detector: Change Detection GLRT (CD-GLRT)}

The target channel responses $\mathbf{H}$ or $\mathbf{H}^{T}$ are assumed to be unknown. The detection problem is

$$
\begin{aligned}
& \mathbb{H}_{1}: \mathbf{u}_{m}=\left[\begin{array}{c}
\mathbf{H} \mathbf{s}_{A} \\
\beta \mathbf{H}^{T} \mathbf{s}_{B}
\end{array}\right]+\left[\begin{array}{c}
\mathbf{v}_{m} \\
\mathbf{w}_{m}
\end{array}\right] \\
& \mathbb{H}_{0}: \mathbf{u}_{m}=\left[\begin{array}{c}
\mathbf{v}_{m} \\
\mathbf{w}_{m}
\end{array}\right] .
\end{aligned}
$$

Again, we use the generalized likelihood ratio test (GLRT), which we refer to as the change detector GLRT (CD-GLRT). Its detailed derivation is in Appendix II. It is given by

$$
\begin{aligned}
\ell_{\mathrm{CD}-\mathrm{GLRT}}(\mathbf{U}) & \\
=\frac{1}{\sigma_{v}^{2} \sigma_{w}^{2}} \sum_{m=1}^{M} & \left\{2 \Re\left\{\beta \sigma_{v}^{2} \mathbf{s}_{B}^{H} \widehat{\mathbf{H}}^{*} \mathbf{r}_{m}+\sigma_{w}^{2} \mathbf{y}_{m}^{T} \hat{\mathbf{H}}^{*} \mathbf{s}_{A}^{*}\right\}\right. \\
& \left.-\sigma_{w}^{2}\left\|\widehat{\mathbf{H}} \mathbf{s}_{A}\right\|^{2}-\beta^{2} \sigma_{v}^{2}\left\|\widehat{\mathbf{H}}^{T} \mathbf{s}_{B}\right\|^{2}\right\}
\end{aligned}
$$

\section{ANALYSIS OF THE IDEAL DETECTORS}

In this section, we evaluate the detection performance of the TRCMF and CDCMF given in (37) and (39), respectively. We derive the closed form expression for the threshold $\eta$ given a false alarm rate $P_{\mathrm{FA}}$ and the probability of detection $P_{\mathrm{D}}$. We will resort to numerical methods to evaluate the performance of the TR-GLRT and the CD-GLRT detectors.

\section{A. False Alarm Rate}

The false alarm probability is defined as $P_{\mathrm{FA}}=\operatorname{prob}\{\ell(\cdot) \geq$ $\left.\eta \mid \mathbb{H}_{0}\right\}$, where $\ell(\cdot)$ denotes the test statistics. Under $\mathfrak{H}_{0}$, the test statistics $\ell_{\mathrm{TRCMF}}(\mathbf{X})$ and $\ell_{\mathrm{CDCMF}}(\mathbf{R})$ given by (37) and by (39), respectively, are the same with $\mathbf{x}_{m}=\mathbf{w}_{m}$. The noise vector $\mathbf{w}_{m}$ at the antennas in $A$ is distributed in both cases as in (29). It can be shown that the quantity inside $\mathfrak{R}(\cdot)$ in (37) or (39) under hypothesis $\mathbb{H}_{0}$, i.e., $\mathbf{x}_{m}=\mathbf{w}_{m}$, is a complex random variable distributed as $\mathcal{C N}(0, P M)$. This implies [20]

$$
\ell_{\mathrm{TRCMF}}(\mathbf{X}) \sim \mathcal{N}\left(0, \frac{P M}{2}\right) .
$$

From (43), the false alarm probability $P_{\mathrm{FA}}$ and the threshold are given successively by

$$
\begin{aligned}
P_{\mathrm{FA}} & =\int_{\eta}^{\infty} \frac{1}{\sqrt{2 \pi P M / 2}} e^{-\frac{t^{2}}{2 P M / 2}} d t \\
& =\int_{\frac{\eta}{\sqrt{P M}}}^{\infty} \frac{1}{\sqrt{\pi}} e^{-t^{2}} d t, \eta \in \mathcal{R} \\
& =\frac{1}{2}\left(1-\operatorname{erf}\left(\frac{\eta}{\sqrt{P M}}\right)\right) \\
\eta & =\sqrt{P M} \operatorname{erf}^{-1}\left(1-2 P_{\mathrm{FA}}\right)
\end{aligned}
$$

where $\operatorname{erf}(\cdot)$ and $\operatorname{erf}^{-1}(\cdot)$ are the error and the inverse error functions, respectively [23]. Next, we compute the probability of detection for the two detectors TRCMF and CDCMF.

\section{B. Detection Probability}

The probability of detection $P_{\mathrm{D}}$ is defined as $P_{\mathrm{D}}=$ $\operatorname{Prob}\left\{\ell(\cdot) \geq \eta \mid \mathbb{H}_{1}\right\}$, where $\ell(\cdot)$ is the test statistic. We first compute $P_{\mathrm{D}}$ for the TRCMF detector.

Time-Reversal Channel Matched Filter (TRCMF). Under hypothesis $\mathbb{H}_{1}$, the test statistic (37) is

$$
\ell_{\mathrm{TRCMF}}(\mathbf{X})=d_{\mathrm{TRCMF}}+\mathfrak{R}\left(\sum_{m=1}^{M} \frac{\left(\mathbf{H}^{T} \mathbf{H}^{*} \mathbf{s}_{A}^{*}\right) \mathbf{w}_{m}}{\sigma_{w}\left\|\mathbf{H}^{T} \mathbf{H}^{*} \mathbf{s}_{A}^{*}\right\|}\right)
$$

where

$$
d_{\mathrm{TRCMF}}=\frac{M k}{\sigma_{w}}\left\|\mathbf{H}^{T} \mathbf{H}^{*} \mathbf{s}_{A}^{*}\right\| .
$$

Again, the noise vector $\mathbf{w}_{m}$ is distributed as in (29), from which it follows that, under $\mathfrak{H}_{1}$,

$$
\ell_{\mathrm{TRCMF}}(\mathbf{X}) \sim \mathcal{N}\left(d_{\mathrm{TRCMF}}, \frac{P M}{2}\right)
$$

which yields the detection probability

$$
\begin{aligned}
P_{\mathrm{D}, \mathrm{TRCMF}}=\int_{\eta_{\mathrm{TRCMF}}}^{\infty} & \frac{1}{\sqrt{2 \pi P M / 2}} \\
& \times \exp \left\{-\frac{\left(t-d_{\mathrm{TRCMF}}\right)^{2}}{2 P M / 2}\right\} d t
\end{aligned}
$$

where $\eta_{\mathrm{TRCMF}}$ is the detection threshold in (45). Making use of the error function

$$
P_{\mathrm{D}, \mathrm{TRCMF}}=\frac{1}{2}\left(1-\operatorname{erf}\left(\frac{\eta_{\mathrm{TRCMF}}-d_{\mathrm{TRCMF}}}{\sqrt{P M}}\right)\right) .
$$

Change Detection Channel Matched Filter (CDCMF). Under hypothesis $\mathbb{H}_{1}$, the decision statistic (39) for the matched filter is given by

$$
\ell_{\mathrm{CDCMF}}(\mathbf{R})=d_{\mathrm{CDCMF}}+\mathfrak{R}\left(\sum_{m=1}^{M} \frac{\left(\mathbf{H}^{T} \mathbf{s}_{B}\right)^{H} \mathbf{w}_{m}}{\sigma_{w}\left\|\mathbf{H}^{T} \mathbf{s}_{B}\right\|}\right)
$$

where

$$
d_{\mathrm{CDCMF}}=\frac{\beta M}{\sigma_{w}}\left\|\mathbf{H}^{T} \mathbf{s}_{B}\right\|
$$

Again, from the complex Gauss statistics of $\mathbf{w}_{m}$, we obtain

$$
\ell_{\mathrm{CDCMF}}(\mathbf{R}) \sim \mathcal{C N}\left(d_{\mathrm{CDCMF}}, \frac{P M}{2}\right) .
$$


The detection probability for CDCMF given the threshold is

$$
P_{\mathrm{D}, \mathrm{CDCMF}}=\frac{1}{2}\left(1-\operatorname{erf}\left(\frac{\eta_{\mathrm{CDCMF}}-d_{\mathrm{CDCMF}}}{\sqrt{P M}}\right)\right) \text {. }
$$

\section{TR AND OPTIMAL WAVEFORM TRANSMISSION}

In this section, we show that TR is an adaptive waveform transmission scheme. We can establish a direct parallel with some conclusions drawn from the optimal radar literature (see, e.g., [24]-[28]), namely, with the design of the transmission waveform to maximize the output SNR at the radar receiver [24]. Our proposed TR-GLRT is different from the waveform preconditioning proposed in [29] that focuses on clutter rejection when the clutter characteristics are known. In this paper, we reject the clutter by direct subtraction and focus on analyzing the impact of the residual clutter and additive noise on detection performance using the generalized-likelihood principle.

We show that TR maximizes the transmitted SNR. Let $\mathbf{f}\left(\omega_{q}\right)$, $\mathbf{H}\left(\omega_{q}\right)$, and $\mathbf{b}\left(\omega_{q}\right)$ denote the vector representations of the transmission waveform, the channel, and the receiver filter response; and $S_{n n}\left(\omega_{q}\right)$ be the noise power spectrum. The optimal matched filter at the receiver is

$$
\mathbf{b}\left(\omega_{q}\right)=\alpha \frac{\left[\mathbf{H}^{T}\left(\omega_{q}\right) \mathbf{f}\left(\omega_{q}\right)\right]^{*}}{\sqrt{S_{n n}\left(\omega_{q}\right)}}, \alpha \in \mathcal{R} .
$$

The output SNR at the receiver is

$$
\begin{aligned}
\mathrm{SNR}_{o} & =\sum_{q=0}^{Q-1} \frac{\left|\mathbf{b}^{H}\left(\omega_{q}\right)\left(\mathbf{H}^{T}\left(\omega_{q}\right) \mathbf{f}\left(\omega_{q}\right)\right)\right|^{2}}{S_{n n}\left(\omega_{q}\right)} \\
& =\alpha^{2} \sum_{q=0}^{Q-1} \frac{\left\|\mathbf{H}^{T}\left(\omega_{q}\right) \mathbf{f}\left(\omega_{q}\right)\right\|^{4}}{S_{n n}\left(\omega_{q}\right)} \\
& =\alpha^{2} \sum_{q=0}^{Q-1}\left|\mathbf{f}^{H}\left(\omega_{q}\right) \mathbf{H}^{*}\left(\omega_{q}\right) \mathbf{H}^{T}\left(\omega_{q}\right) \mathbf{f}\left(\omega_{q}\right)\right|^{2} \\
& =\alpha^{2} \sum_{q=0}^{Q-1}\left|\mathbf{f}^{H}\left(\omega_{q}\right) \mathbf{K}\left(\omega_{q}\right) \mathbf{f}\left(\omega_{q}\right)\right|^{2} \\
& =\alpha^{2}\left\|\mathbf{f}^{H} \mathbf{K f}\right\|^{2}
\end{aligned}
$$

where for simplicity, we assume that $S_{n n}\left(\omega_{q}\right)=\sigma_{v}^{2}, \forall q$, and the Hermitian matrix $\mathbf{K}$

$$
\begin{aligned}
\mathbf{K} & =\operatorname{diag}\left[\mathbf{K}\left(\omega_{0}\right), \ldots, \mathbf{K}\left(\omega_{Q-1}\right)\right] \\
\mathbf{K}\left(\omega_{q}\right) & =\mathbf{H}^{*}\left(\omega_{q}\right) \mathbf{H}^{T}\left(\omega_{q}\right)
\end{aligned}
$$

is the TR matrix. The waveform vector is

$$
\mathbf{f}=\left[\mathbf{f}^{T}\left(\omega_{0}\right), \ldots, \mathbf{f}^{T}\left(\omega_{Q-1}\right)\right]^{T} .
$$

Next, we introduce a Green's function representation of $\mathbf{H}\left(\omega_{q}\right)$. Let $G\left(\mathbf{r}, \mathbf{x} ; \omega_{q}\right)$ be the Green's function between locations $\mathbf{x}$ and $\mathbf{r}$ at frequency $\omega_{q}$. It represents the channel (or medium) transfer function from a source at $\mathbf{x}$ and an antenna at $\mathbf{r}$. The Green's function vector responses are

$$
\begin{aligned}
& \mathbf{g}_{B}\left(\omega_{q}\right)=\left[G\left(\mathbf{r}_{B_{1}}, \mathbf{x} ; \omega_{q}\right), \ldots, G\left(\mathbf{r}_{B_{N}}, \mathbf{x} ; \omega_{q}\right)\right]_{N \times 1}^{T} \\
& \mathbf{g}_{A}\left(\omega_{q}\right)=\left[G\left(\mathbf{r}_{A_{1}}, \mathbf{x} ; \omega_{q}\right), \ldots, G\left(\mathbf{r}_{A_{P}}, \mathbf{x} ; \omega_{q}\right)\right]_{P \times 1}^{T} .
\end{aligned}
$$

We decompose the channel matrix as [11]

$$
\mathbf{H}\left(\omega_{q}\right)=\tau \mathbf{g}_{B}\left(\omega_{q}\right) \mathbf{g}_{A}^{T}\left(\omega_{q}\right)
$$

Let the target and antenna locations be denoted by $\mathbf{x}$ and $\mathbf{r}_{B_{n}}$ (or $\mathbf{r}_{A_{p}}$ ), respectively, and $\tau$ be the target reflectivity. Each $G\left(\mathbf{r}, \mathbf{x} ; \omega_{q}\right)$ represents the medium Green's function induced by the presence of the target. The TR matrix is rewritten as

$$
\mathbf{K}\left(\omega_{q}\right)=|\tau|^{2} \mathbf{g}_{B}^{*}\left(\omega_{q}\right) \mathbf{g}_{B}^{T}\left(\omega_{q}\right)\left\|\mathbf{g}_{A}\left(\omega_{q}\right)\right\|^{2} .
$$

The waveform design problem chooses $\mathbf{f}$ such that

$$
\mathbf{f}_{\mathrm{opt}}=\arg \max _{\mathbf{f}}\left(\mathrm{SNR}_{o}\right)
$$

subject to the constraint

$$
\frac{1}{Q}\|\mathbf{f}\|^{2}=E_{f}
$$

where $E_{f}$ is the total transmission energy of the waveform $\mathrm{f}_{\text {opt }}$. In (66), the output SNR $\left(\mathrm{SNR}_{o}\right)$ is given in (56). The optimal $\mathbf{f}_{\mathrm{opt}}\left(\omega_{q}\right)$ at $\omega_{q}$ is given by the eigenvector of $\mathbf{K}\left(\omega_{q}\right)$ corresponding to the largest eigenvalue, [30]. Since $\mathbf{K}\left(\omega_{q}\right)$ given in (65) is a rank one matrix, the optimal solution is

$$
\mathbf{f}_{\mathrm{opt}}\left(\omega_{q}\right)=\gamma \mathbf{g}_{B}^{*}\left(\omega_{q}\right)
$$

where $\gamma$ is a complex scalar. Comparing (4) and (68), we make the following observations.

i) Assuming that the noise in the time-reversed signal is negligible, $S_{n n}\left(\omega_{q}\right)=\sigma_{v}^{2}, \forall q$, and adopting the vector Green's function representation of $\mathbf{H}\left(\omega_{q}\right)$ in (64), the time reversed signal in (4) is, up to a constant scaling factor, exactly the optimal transmission waveform $\mathbf{f}\left(\omega_{q}\right)$ in (68), i.e.,

$$
\begin{aligned}
\mathbf{y}_{m}^{*}\left(\omega_{q}\right) & =\left[\mathbf{H}\left(\omega_{q}\right) \mathbf{s}_{A}\left(\omega_{q}\right)\right]^{*} \\
& =\lambda\left(\omega_{q}\right) \mathbf{g}_{B}^{*}\left(\omega_{q}\right)
\end{aligned}
$$

where

$$
\lambda\left(\omega_{q}\right)=\sum_{i=1}^{P} G\left(\mathbf{r}_{P_{i}}, \mathbf{x} ; \omega_{q}\right) S\left(\omega_{q}\right)
$$

is a complex scalar. Thus, the TR transmission scheme maximizes the output $\mathrm{SNR}, \mathrm{SNR}_{o}$, at the receiver. This result (70) shows that the TR operation reconstructs the Green's function vector $\mathbf{g}_{B}\left(\omega_{q}\right)$ between the target and the array and thus adaptively adjusts the transmission waveform to the channel characteristics.

ii) When the noise contained in the time-reversed waveform is not negligible, the TR transmission is an approximation to the optimal transmission strategy. Part of the 
transmission power is wasted due to the noise present in the time reversed signals. The impact of the noise component in the time-reversed signal will be discussed in Section VI-B.

\section{SNR GAIN IN TR DETECTION}

This section develops closed forms for the SNR detection gains afforded by TR over conventional detection using antenna arrays, where we define the performance comparison as the difference of likelihood ratios [21]. We compute two gains: the maximal SNR gain $\left(\mathrm{SNRG}_{\max }\right)$ achievable by TRCMF over CDCMF computed by assuming that the noise in the time reversed retransmitted signal is negligible; and the asymptotic SNR gain $\left(\mathrm{SNRG}_{\mathrm{asym}}\right)$ that quantifies the performance gain of TR TR-GLRT (asym) over CD-GLRT $_{(\text {asym) }}$ when the noise in the retransmitted time reversed signal is taken into account. To simplify the analysis, we assume that the target channel response $\mathbf{H}\left(\omega_{q}\right), \forall q$, is known. (Note that, in this case, CD-GLRT $_{(\text {asym) }}$ is equivalent to CDCMF.)

\section{A. Maximal SNR Gain of TRCMF $\left(\mathrm{SNRG}_{\max }\right)$}

We evaluate the performance gain provided by time reversal (TRCMF) over conventional detection (CDCMF) for a fixed false alarm rate. We take $\sigma_{v}^{2}=\sigma_{w}^{2}$. The maximal TR SNR detection gain $\left(\mathrm{SNRG}_{\max }\right)$ is given by the ratio of $d_{\mathrm{TRCMF}}^{2}$ to $d_{\mathrm{CDCMF}}^{2}$ given in (47) and (52), i.e.,

$$
\begin{aligned}
\mathrm{SNRG}_{\max } & =\frac{d_{\mathrm{TRCMF}}^{2}}{d_{\mathrm{CDCMF}}^{2}} \\
& =\frac{k^{2}\left\|\mathbf{H}^{T} \mathbf{H}^{*} \mathbf{s}_{A}^{*}\right\|^{2}}{\beta^{2}\left\|\mathbf{H}^{T} \mathbf{s}_{B}\right\|^{2}} \\
& =\frac{\left\|\mathbf{s}_{A}\right\|^{2}\left\|\mathbf{H}^{T} \mathbf{H}^{*} \mathbf{s}_{A}^{*}\right\|^{2}}{(P / N)\left\|\mathbf{H}^{T} \mathbf{s}_{B}\right\|^{2}\left\|\mathbf{H} \mathbf{s}_{A}\right\|^{2}} .
\end{aligned}
$$

The constant gains $k$ and $\beta$ are given in (35) and (25), respectively. This expression generalizes the maximal gain expression (see (89) in [2]) when $P=N=1$.

The gain in (71) varies depending on the array configuration and the variations of the target response $\mathbf{H}\left(\omega_{q}\right)$ across the frequency range. To gain insight, we study two simple cases as we trade off between $N, P$, and $Q$. We resort to numerical means to study a more general case with arbitrary $P, N$, and $Q$. For simplicity, assume that

$$
\begin{aligned}
\mathbf{s}_{A}\left(\omega_{q}\right) & =S\left(\omega_{q}\right) \mathbf{1}_{P}, \mathbf{s}_{B}\left(\omega_{q}\right) \\
& =S\left(\omega_{q}\right) \mathbf{1}_{N}, \text { and } S\left(\omega_{q}\right)=1, \forall q .
\end{aligned}
$$

1) Case-1, $P=N=1$ : This is when we have a single antenna pair. Reference [2] shows that $\mathrm{SNRG} \geq 1$. Equality holds when the amplitude of the channel response is flat. A large gain can be achieved if the target channel response across frequencies, i.e., the channel type, varies significantly.

2) Case-2, $P=1, N>1$ : This is called TR mirror (TRM) in acoustics [3], [31] and in electromagnetics [14], [32]. For TRM, a single probe source illuminates the target area and the scattered field is recorded by an array of sensors. The array timereverses the signal and sends it back to the target area. This focuses the scattered field at the location of the probe source.
We study the type or empirical distribution of the channel. The channel matrix is

$$
\mathbf{H}\left(\omega_{q}\right)=\mathbf{h}\left(\omega_{q}\right)=\left[h_{1}\left(\omega_{q}\right), h_{2}\left(\omega_{q}\right), \ldots, h_{N}\left(\omega_{q}\right)\right]^{T}
$$

where $h_{n}\left(\omega_{q}\right), 1 \leq n \leq N$, are the frequency responses of the channel between the transmit antenna $A_{1}$ and the receive antennas $B_{n}, 1 \leq n \leq N$. The gain is

$$
\begin{array}{r}
\mathrm{SNRG}_{\max }=2 \frac{\frac{1}{Q} \sum_{q=0}^{Q-1}\left(\left|h_{1}\left(\omega_{q}\right)\right|^{2}+\left|h_{2}\left(\omega_{q}\right)\right|^{2}\right)^{2}}{\frac{1}{Q} \sum_{q=0}^{Q-1}\left(\left|h_{1}\left(\omega_{q}\right)\right|^{2}+\left|h_{2}\left(\omega_{q}\right)\right|^{2}\right)} \\
\times \frac{1}{\frac{1}{Q} \sum_{q=0}^{Q-1}\left|h_{1}\left(\omega_{q}\right)+h_{2}\left(\omega_{q}\right)\right|^{2}}
\end{array}
$$

In a rich scattering environment, the channel bandwidth is described by the coherence bandwidth [19], $B_{c}$, which is given approximately by the inverse of the largest channel delay spread. For a signal with bandwidth BW, the number of uncorrelated frequency samples is then approximately given by

$$
Q_{0}=\frac{B W}{B_{c}} \text {. }
$$

This is because if we take data samples at frequencies $B_{c} \mathrm{~Hz}$ apart, we obtain practically independent information about the scattering characteristics of the channel [19]. The richer the scattering is, the smaller the coherence bandwidth of the channel is, leading to larger number of available uncorrelated frequency samples. Choosing $Q<Q_{0}$ implies that the channel is undersampled; while $Q>Q_{0}$ means the channel is over-sampled. Next, we examine the SNR gain in (73) when the signal is narrowband $(Q=1)$ and wideband $(Q>1)$, respectively.

i) Narrowband $Q=1, \omega_{q}=\omega$ : In this case, the signal bandwidth is smaller than the coherence bandwidth of the channel. The TR mirror (TRM) becomes a transmit beamformer with weight vector

$$
\mathbf{g}=\left[S^{*}(\omega) h_{1}^{*}(\omega), S^{*}(\omega) h_{2}^{*}(\omega)\right]^{T}
$$

while the conventional detector illuminates the scattering field with a uniform probing signal $S(\omega)$. The gain expression in (73) becomes

$$
\mathrm{SNRG}_{\max }=2 \frac{\left|h_{1}(\omega)\right|^{2}+\left|h_{2}(\omega)\right|^{2}}{\left|h_{1}(\omega)+h_{2}(\omega)\right|^{2}} \geq 1 .
$$

Equality holds when $h_{1}(\omega)=h_{2}(\omega)$. With the conventional method, the probing signals transmitted from the two antennas can result in a null at the receiver location when $h_{1}(\omega)+h_{2}(\omega)=0$, while the TRM removes the phase term from the received signal, thus not creating a null.

ii) Wideband $Q \gg 1$ : The signal bandwidth is much larger than the coherence bandwidth of the channel, $Q$ is a large number. By considering the empirical distribution (or type [1]) for $h_{1}\left(\omega_{q}\right)$ and $h_{2}\left(\omega_{q}\right)$, i.e., the normalized histogram of the target channel responses over $Q$ frequencies, we can further analyze the potential SNR gain. In the high frequency range, the phases of the frequency samples $h_{1}\left(\omega_{q}\right)$ or $h_{2}\left(\omega_{q}\right)$ change rapidly between $[0,2 \pi]$. To get quantitative results, we approximate the 
empirical distribution by the normal distribution. This computation uses the common practice of modeling the channel $h_{1}$ and $h_{2}$ as independent complex normal variables [19], $h_{1}, h_{2} \sim \mathcal{C N}(0, \gamma)$, i.e., the multipath Rayleigh model. ${ }^{1}$ For simplicity, we take $h_{1}$ and $h_{2}$ with the same variance. Thus, the magnitude $\left|h_{1}\right|$ and $\left|h_{2}\right|$ are Rayleigh distributed. Let $\chi_{\sigma}$ denote a Rayleigh random variable with $\sigma$ degrees of freedom. The $r$ th raw moment of $\chi_{\sigma}$, as well as the first few raw moments are [33]

$$
\begin{aligned}
\mu_{r}^{\prime} & =E\left[\chi_{\sigma}^{r}\right]=\sigma^{r} 2^{r / 2} \Gamma\left(\frac{r}{2}+1\right) \\
E\left\{\left|h_{1}\right|\right\} & =\frac{\sqrt{\pi}}{2} \sqrt{\gamma}, E\left\{\left|h_{1}\right|^{2}\right\}=\gamma, \text { and } E\left\{\left|h_{1}\right|^{4}\right\}=2 \gamma^{2}
\end{aligned}
$$

where $\Gamma(\cdot)$ is the Gamma function [23]; in our case, $\sigma=\sqrt{\gamma / 2}$. Hence, an equivalent statistical expression of (73) is [see (78) and (79), shown at the bottom of the page]. Equation (79) is a function of the cross correlation of the channel frequency samples $h_{1}$ and $h_{2}$ measured at the two antennas. The cross correlation depends on the relative distance of the two antennas, as well as the characteristics of the scattering field. A large interelement spacing between the two antennas, for example, a few wavelengths apart, in a rich multipath scattering implies that the cross correlation of the two spatial channels is small, i.e., the two channels fade independently. In this case, the two random variables $h_{1}$ and $h_{2}$ are independent, and we have

$$
E\left\{\left|h_{1}\right|^{2}\left|h_{2}\right|^{2}\right\}=E\left\{\left|h_{1}\right|^{2}\right\} E\left\{\left|h_{2}\right|^{2}\right\}=\gamma^{2}
$$

and $E\left\{h_{1} h_{2}^{*}\right\}=E\left\{h_{1}\right\} E\left\{h_{2}^{*}\right\}=0$, which yields for the Rayleigh channel fading model

$$
\mathrm{SNRG}_{\max }=2 \frac{4 \gamma^{2}+2 \gamma^{2}}{2 \gamma 2 \gamma}=3=4.7 \mathrm{~dB} .
$$

Equation (80) implies that for the Rayleigh channel when the antennas are placed sparsely [14], [32], the TR mirror (TRM) detector can lead to, on average, a 4.7-dB gain over the conventional change detection in a rich Rayleigh multipath. We conduct numerical simulations to verify the analysis. The top panel

\footnotetext{
${ }^{1}$ The corresponding analysis can also be extended to a general Rician model, or, depending on the channel empirical distribution, to other more complicated statistical models.
}
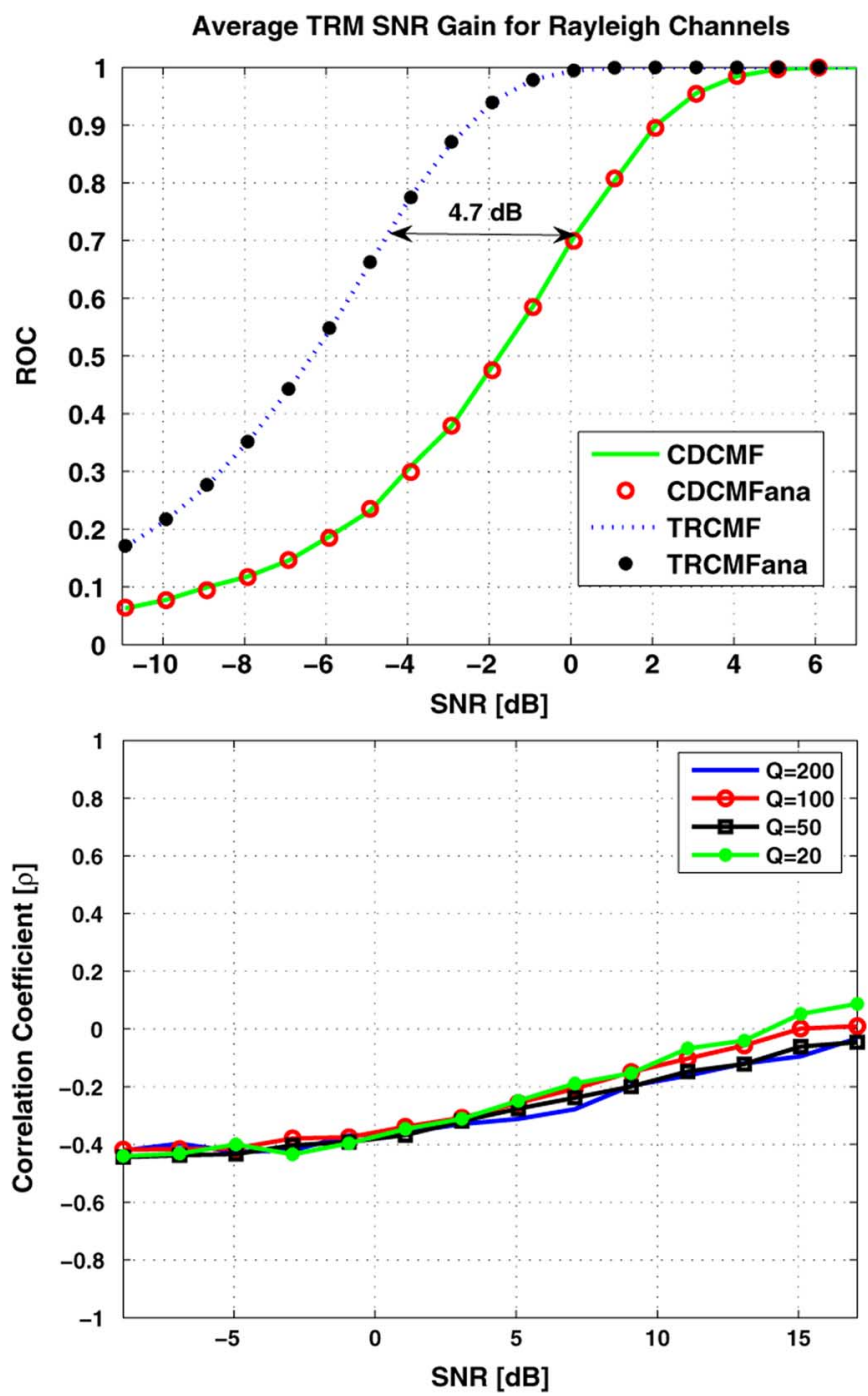

Fig. 1. Top panel: ROC for Rayleigh channels. The distance between the two ROC plots is the SNR gain of the TR Mirror given in (80). The ROC plots are generated over 20 runs. $P_{\mathrm{fa}}=0.01$. Bottom panel: simulation verification for (104) by computing the Pearson's correlation coefficient $\rho$.

in Fig. 1 shows the SNR gain by TRM detection using two antennas. The two channels are characterized by Rayleigh models. The ROC plot shows that TRM has a 4.7-dB gain over the conventional method.

Transmit Array Gain by the TR Mirror: Using the analysis shown above, we can characterize the transmit array gain [34], [35] induced by the TRM. We show that a TRM of $N$-antennas yields a transmit array gain greater than the normal array gain

$$
\begin{aligned}
\mathrm{SNRG}_{\max } & =2 \frac{E\left\{\left(\left|h_{1}\right|^{2}+\left|h_{2}\right|^{2}\right)^{2}\right\}}{E\left\{\left|h_{1}\right|^{2}+\left|h_{2}\right|^{2}\right\} E\left\{\left|h_{1}+h_{2}\right|^{2}\right\}} \\
& =2 \frac{E\left\{\left|h_{1}\right|^{4}\right\}+E\left\{\left|h_{2}\right|^{4}\right\}+2 E\left\{\left|h_{1}\right|^{2}\left|h_{2}\right|^{2}\right\}}{\left(E\left\{\left|h_{1}\right|^{2}\right\}+E\left\{\left|h_{2}\right|^{2}\right\}\right)\left(E\left\{\left|h_{1}\right|^{2}\right\}+E\left\{\left|h_{2}\right|^{2}\right\}+2 \mathfrak{R}\left(E\left\{h_{1} h_{2}^{*}\right\}\right)\right)} \\
& =2 \frac{4 \gamma^{2}+2 E\left\{\left|h_{1}\right|^{2}\left|h_{2}\right|^{2}\right\}}{2 \gamma\left(2 \gamma+2 \mathfrak{R}\left(E\left\{h_{1} h_{2}^{*}\right\}\right)\right)} .
\end{aligned}
$$


of $N$. Again, we use the $P=1, N=2$ channel model given in (72). We consider the ideal scenario where the noise is white spatially across the array and has a flat power spectrum. Under these conditions, (47) is the output SNR for the TRCMF. We compare the output SNR of the TRCMF, $d_{\mathrm{TRCMF}}^{2}$ in (47), for the channel (72) when $P=1, N=2$, with the output SNR, $d_{2}^{2}$, given by (61) of [2], for the channel $h_{1}\left(\omega_{q}\right)$ when $P=1, N=$ 1 . Hence, we define

$$
A_{g}^{\mathrm{TRM}} \triangleq \frac{\frac{\left\|\mathbf{s}_{A}\right\|^{2}\left\|\mathbf{H}^{T} \mathbf{H}^{*} \mathbf{s}_{A}^{*}\right\|^{2}}{\left\|\mathbf{H} \mathbf{s}_{A}\right\|^{2}}}{\frac{\sum_{q=0}^{Q-1}\left|S\left(\omega_{q}\right)\right|^{2} \sum_{q=0}^{Q-1}\left|h_{1}\left(\omega_{q}\right)\right|^{4}\left|S\left(\omega_{q}\right)\right|^{2}}{\sum_{q=0}^{Q-1}\left|h_{1}\left(\omega_{q}\right)\right|^{2}\left|S\left(\omega_{q}\right)\right|^{2}}} .
$$

Assuming that $S\left(\omega_{q}\right)=1, \forall q$, we rewrite (81) as

$$
A_{g}^{\mathrm{TRM}}=\frac{2 \sum_{q=0}^{Q-1}\left(\left|h_{1}\left(\omega_{q}\right)\right|^{2}+\left|h_{2}\left(\omega_{q}\right)\right|^{2}\right)^{2}}{\sum_{q=0}^{Q-1}\left|h_{1}\left(\omega_{q}\right)+h_{2}\left(\omega_{q}\right)\right|^{2}} \frac{\sum_{q=0}^{Q-1}\left|h_{1}\left(\omega_{q}\right)\right|^{2}}{\sum_{q=0}^{Q-1}\left|h_{1}\left(\omega_{q}\right)\right|^{4}} .
$$

Similar to the analysis in (79), we obtain

$$
\begin{aligned}
A_{g}^{\mathrm{TRM}} & =2 \frac{4 \gamma^{2}+2 E\left\{\left|h_{1}\right|^{2}\left|h_{2}\right|^{2}\right\}}{2 \gamma\left(2 \gamma+2 \Re\left\{E\left(h_{1} h_{2}^{*}\right)\right\}\right)} \\
& =2 \frac{4 \gamma^{2}+2 \gamma^{2}}{2 \gamma 2 \gamma} \\
& =3=4.7 \mathrm{~dB}
\end{aligned}
$$

where we assume that $h_{1}$ and $h_{2}$ are independent fading Rayleigh channel coefficients. We conclude that, under rich scattering Rayleigh channel models, a 2-antenna TRM results in a transmit array gain greater than the nominal array gain of 2 (or $3 \mathrm{~dB}$ ). The explanation is intuitive: TRM adaptively matches to the target channel response, optimally exploiting the multipath diversity and the spatial diversity. The multipath diversity is caused by the rich multipath scattering that induces a channel response that is frequency dependent; the spatial diversity arises due to the large element spacing, resulting in independent channel responses for each antenna of the TRM array.

\section{B. Asymptotic SNR Gain of TR-GLRT (SNRG asym $\left._{\text {S }}\right)$}

In physical TR, the signal received at the antennas of array $B$ after the initial transmission from the antennas at array $A$ is corrupted by additive noise. When this signal is time reversed and retransmitted, a fraction of the transmission power is wasted on the noise component. In this section, we quantify the performance gain of TR over the conventional detection when the additive noise is taken into account. For simplicity, we assume that the target frequency response is known. We emphasize that, in the following analysis, the detection problems under study are slightly different from (40) and (42) since now the target response $\mathbf{H}\left(\omega_{q}\right)$ is assumed to be known in both cases. Thus, the results we derive correspond to an asymptotic performance gain of TR detection over conventional detection (in the limiting case of high SNR or many samples so that the channel response becomes asymptotically known).
Given (13) and (4), the optimal detector is the likelihood ratio of the joint pdf of $\left(\mathbf{y}_{m}\left(\omega_{q}\right), \mathbf{x}_{m}\left(\omega_{q}\right)\right)$,

$$
\begin{aligned}
& \ell_{\mathrm{TR}-\mathrm{GLRT}(\text { asym) }} \\
& =\ln \frac{\prod_{m=1}^{M} \prod_{q=0}^{Q-1} p_{1}\left(\mathbf{x}_{m}\left(\omega_{q}\right), \mathbf{y}_{m}\left(\omega_{q}\right)\right)}{\prod_{m=1}^{M} \prod_{q=0}^{Q-1} p_{0}\left(\mathbf{x}_{m}\left(\omega_{q}\right), \mathbf{y}_{m}\left(\omega_{q}\right)\right)} \\
& =\ln \frac{\prod_{m=1}^{M} \prod_{q=0}^{Q-1} p_{1}\left(\mathbf{x}_{m}\left(\omega_{q}\right) \mid \mathbf{y}_{m}\left(\omega_{q}\right)\right)}{\prod_{m=1}^{M} \prod_{q=0}^{Q-1} p_{0}\left(\mathbf{x}_{m}\left(\omega_{q}\right) \mid \mathbf{y}_{m}\left(\omega_{q}\right)\right)} \\
& +\ln \frac{\prod_{m=1}^{M} \prod_{q=0}^{Q-1} p_{1}\left(\mathbf{y}_{m}\left(\omega_{q}\right)\right)}{\prod_{m=1}^{M} \prod_{q=0}^{Q-1} p_{0}\left(\mathbf{y}_{m}\left(\omega_{q}\right)\right)}
\end{aligned}
$$

The $p_{i}\left(\mathbf{x}_{m}\left(\omega_{q}\right) \mid \mathbf{y}_{m}\left(\omega_{q}\right)\right)$ is the conditional probability density function for $\mathbf{x}_{m}\left(\omega_{q}\right)$ given $\mathbf{y}_{m}\left(\omega_{q}\right)$ under $\mathbb{H}_{i}, i=0,1$.

Similarly, the optimal conventional detector assuming $\mathbf{H}\left(\omega_{q}\right), \forall q$, known is the likelihood ratio of the joint pdf of the measurement pair $\left(\mathbf{y}_{m}, \mathbf{r}_{m}\right)$. It leads to

$$
\begin{aligned}
\ell_{\mathrm{CD}-\mathrm{GLRT}(\operatorname{asym})=\ln } \frac{\prod_{m=1}^{M} \prod_{q=0}^{Q-1} p_{1}\left(\mathbf{r}_{m}\left(\omega_{q}\right)\right)}{\prod_{m=1}^{M} \prod_{q=0}^{Q-1} p_{0}\left(\mathbf{r}_{m}\left(\omega_{q}\right)\right)} \\
+\ln \frac{\prod_{m=1}^{M} \prod_{q=0}^{Q-1} p_{1}\left(\mathbf{y}_{m}\left(\omega_{q}\right)\right)}{\prod_{m=1}^{M} \prod_{q=0}^{Q-1} p_{0}\left(\mathbf{y}_{m}\left(\omega_{q}\right)\right)} .
\end{aligned}
$$

The test statistic of the detector CD-GLRT $($ asym) given in (85) is equivalent to CDCMF when the target channel is known, i.e., $\ell_{\mathrm{CD}-\mathrm{GLRT} \text { (asym) }}=\ell_{\mathrm{CDCMF}}$. Next, define the performance comparison between TR-GLRT (asym) $_{\text {and CD-GLRT }}$ (asym) as the difference of the likelihood ratios defined in (84) and (85), respectively. In (84) and (85), the second term is common, and so this term does not affect the performance comparison between (84) and (85). Therefore, it suffices to examine the remaining terms. These terms are complex Gaussian distributed with a nonzero mean, i.e., the first term in (84) and the first term in (85) are distributed as $\mathcal{C N}\left(\mathbf{x}\left(\omega_{q}\right), \sigma_{w}^{2}\right)$ and $\mathcal{C N}\left(\mathbf{r}\left(\omega_{q}\right), \sigma_{w}^{2}\right)$, respectively, at frequency $\omega_{q}$, where the means are given as follows:

$$
\begin{aligned}
\mathbf{x}\left(\omega_{q}\right) & =k_{m} \mathbf{H}^{T}\left(\omega_{q}\right) \mathbf{y}^{*}\left(\omega_{q}\right), \text { for }(84) \\
\mathbf{r}\left(\omega_{q}\right) & =\beta \mathbf{H}\left(\omega_{q}\right) \mathbf{s}_{B}\left(\omega_{q}\right), \text { for }(85) .
\end{aligned}
$$

Thus, for a given $m$, the performance comparison leads to

$$
\begin{aligned}
d_{\mathrm{TR}-\mathrm{GLRT}(\text { asym })}^{m} & =\frac{\sum_{q=0}^{Q-1}\left\|\mathbf{x}\left(\omega_{q}\right)\right\|^{2}}{Q \sigma_{w}^{2}} \\
& =\frac{k_{m}^{2}}{Q \sigma_{w}^{2}} \sum_{q=0}^{Q-1}\left\|\mathbf{H}^{T}\left(\omega_{q}\right) \mathbf{y}_{m}^{*}\left(\omega_{q}\right)\right\|^{2} \\
& =\frac{k_{m}^{2}}{Q \sigma_{w}^{2}}\left\|\mathbf{H}^{T} \mathbf{y}_{m}^{*}\right\|^{2} \\
d_{\mathrm{CD}-\mathrm{GLRT}(\mathrm{asym})}^{m} & =\frac{\sum_{q=0}^{Q-1}\left\|\mathbf{r}\left(\omega_{q}\right)\right\|^{2}}{Q \sigma_{w}^{2}} \\
& =\frac{\beta^{2}}{Q \sigma_{w}^{2}} \sum_{q=0}^{Q-1}\left\|\mathbf{H}^{T}\left(\omega_{q}\right) \mathbf{s}_{B}\left(\omega_{q}\right)\right\|^{2} \\
& =\frac{\beta^{2}}{Q \sigma_{w}^{2}}\left\|\mathbf{H}^{T} \mathbf{s}_{B}\right\|^{2} .
\end{aligned}
$$


We define the average SNR gain of TR - GLRT (asym) $_{\text {as }}$ as the ratio of the expectation of these two quantities. Using (35), we obtain

$$
\begin{aligned}
\mathrm{SNRG}_{\text {asym }} & \triangleq E\left\{\frac{\sum_{m=1}^{M} d_{\mathrm{TR}-\mathrm{GLRT}(\text { asym })}^{m}}{\sum_{m=1}^{M} d_{\mathrm{CD}-\mathrm{GLRT}(\text { asym })}^{m}}\right\} \\
& =\frac{(N / P) \sum_{q=0}^{Q-1}\left\|\mathbf{s}_{A}\left(\omega_{q}\right)\right\|^{2}}{\sum_{q=0}^{Q-1}\left\|\mathbf{H}^{T}\left(\omega_{q}\right) \mathbf{s}_{B}\left(\omega_{q}\right)\right\|^{2}} E\left\{\frac{\Phi}{\Upsilon}\right\} \\
& =\frac{(N / P)\left\|\mathbf{s}_{A}\right\|^{2}}{\left\|\mathbf{H}^{T} \mathbf{s}_{B}\right\|^{2}} E\left\{\frac{\Phi}{\Upsilon}\right\}
\end{aligned}
$$

where the quantities inside of $E\{\cdot\}$ are

$$
\begin{aligned}
\Phi= & \sum_{q=0}^{Q-1} \| \mathbf{H}^{T}\left(\omega_{q}\right) \mathbf{H}^{*}\left(\omega_{q}\right) \mathbf{s}_{A}^{*}\left(\omega_{q}\right) \\
& +\mathbf{H}^{T}\left(\omega_{q}\right) \mathbf{v}_{m}^{*}\left(\omega_{q}\right)\left\|^{2}=\right\| \mathbf{H}^{T} \mathbf{H}^{*} \mathbf{s}_{A}^{*}+\mathbf{H}^{T} \mathbf{v}_{m}^{*} \|^{2} \\
\Upsilon= & \sum_{q=0}^{Q-1}\left\|\mathbf{H}\left(\omega_{q}\right) \mathbf{s}_{A}\left(\omega_{q}\right)+\mathbf{v}_{m}\left(\omega_{q}\right)\right\|^{2}=\left\|\mathbf{H} \mathbf{s}_{A}+\mathbf{v}_{m}\right\|^{2}
\end{aligned}
$$

To evaluate $\mathrm{SNRG}_{\mathrm{asym}}$, we calculate the statistics of the random variables $\Phi$ and $\Upsilon$. First, we show that

$$
\begin{aligned}
E\{\Phi\} & =\sum_{q=0}^{Q-1}\left\|\mathbf{H}\left(\omega_{q}\right)\right\|^{2} \sigma_{v}^{2}+\sum_{q=0}^{Q-1}\left\|\mathbf{H}^{T}\left(\omega_{q}\right) \mathbf{H}^{*}\left(\omega_{q}\right) \mathbf{s}_{A}^{*}\left(\omega_{q}\right)\right\|^{2} \\
& =\|\mathbf{H}\|^{2} \sigma_{v}^{2}+\left\|\mathbf{H}^{T} \mathbf{H}^{*} \mathbf{s}_{A}^{*}\right\|^{2} .
\end{aligned}
$$

Let $t_{k}\left(\omega_{q}\right)$ denote the $k$ th entry of the vector $\mathbf{H}^{T}\left(\omega_{q}\right) \mathbf{y}_{m}^{*}\left(\omega_{q}\right)$. We have

$$
\Phi=\sum_{q=0}^{Q-1} \sum_{k=1}^{P}\left|t_{k}\left(\omega_{q}\right)\right|^{2} .
$$

We obtain [33]

$$
\begin{aligned}
t_{k}\left(\omega_{q}\right)= & \sum_{j=1}^{P} \sum_{i=1}^{N} h_{i k}\left(\omega_{q}\right) h_{i j}^{*}\left(\omega_{q}\right) S_{j}^{*}\left(\omega_{q}\right) \\
& +\sum_{i=1}^{N} h_{i k}\left(\omega_{q}\right) V_{m, i}^{*}\left(\omega_{q}\right) \\
\sim & \mathcal{C N}\left(\sum_{j=1}^{P} \sum_{i=1}^{N} h_{i k}\left(\omega_{q}\right) h_{i j}^{*}\left(\omega_{q}\right)\right. \\
& \left.\times S_{j}^{*}\left(\omega_{q}\right), \sum_{i=1}^{N}\left|h_{i k}\left(\omega_{q}\right)\right|^{2} \sigma_{v}^{2}\right)
\end{aligned}
$$

where the random variables $V_{m, n}\left(\omega_{q}\right) \sim \mathcal{C N}\left(0, \sigma_{v}^{2}\right)$. Note that $t_{k}\left(\omega_{q}\right)$ is complex normal; we obtain

$$
\begin{aligned}
\left|t_{k}\left(\omega_{q}\right)\right|^{2} & \sim \frac{\sum_{i=1}^{N}\left|h_{i k}\left(\omega_{q}\right)\right|^{2} \sigma_{v}^{2}}{2} \chi_{2}^{2} \\
& \times\left(\frac{\left|\sum_{j=1}^{P} \sum_{i=1}^{N} h_{i k}\left(\omega_{q}\right) h_{i j}^{*}\left(\omega_{q}\right) S_{j}^{*}\left(\omega_{q}\right)\right|^{2}}{\sum_{i=1}^{N}\left|h_{i k}\left(\omega_{q}\right)\right|^{2} \sigma_{v}^{2} / 2}\right)
\end{aligned}
$$

where $\chi_{2}^{2}(d)$ denotes the noncentral $\chi^{2}$-distribution with two degrees of freedom and noncentrality parameter $d$. The two degrees of freedom arise from the real and imaginary parts of $t_{k}\left(\omega_{q}\right)$. Therefore, given (91), (94) is the sum of independent noncentral $\chi^{2}$-random variables with 2 degrees of freedom. From [33], the mean of a noncentral $\chi^{2}$-distributed random variable is the sum of the degrees of freedom and the noncentral parameter. Hence,

$$
\begin{aligned}
E\{\Phi\}= & \sum_{q=0}^{Q-1} \sum_{k=1}^{P} E\left\{\left|t_{k}\left(\omega_{q}\right)\right|^{2}\right\} \\
= & \sum_{q=0}^{Q-1} \sum_{k=1}^{P} \sum_{i=1}^{N}\left|h_{i k}\left(\omega_{q}\right)\right|^{2} \sigma_{v}^{2} \\
& +\sum_{q=0}^{Q-1} \sum_{k=1}^{P}\left|\sum_{j=1}^{P} \sum_{i=1}^{N} h_{i k}\left(\omega_{q}\right) h_{i j}^{*}\left(\omega_{q}\right) S_{j}^{*}\left(\omega_{q}\right)\right|^{2} .
\end{aligned}
$$

Written in a compact form, (97) becomes (93). Next, we obtain the distribution of $\Upsilon$. Let

$$
l_{k}(\omega)=\sum_{j=1}^{P} h_{k j}\left(\omega_{q}\right) S_{j}\left(\omega_{q}\right)+V_{m, k}\left(\omega_{q}\right)
$$

be the $k$ th entry of $\mathbf{H}\left(\omega_{q}\right) \mathbf{s}_{A}\left(\omega_{q}\right)$ in (92). Using the properties of noncentral chi-square distributions for complex numbers, we obtain

$$
\left|l_{k}(\omega)\right|^{2} \sim \frac{\sigma_{v}^{2}}{2} \chi_{2}^{2}\left(\frac{\left|\sum_{j=1}^{P} h_{k j}\left(\omega_{q}\right) S_{j}\left(\omega_{q}\right)\right|^{2}}{\sigma_{v}^{2} / 2}\right) .
$$

It is straightforward to obtain

$$
\begin{aligned}
\Upsilon & =\sum_{q=0}^{Q-1} \sum_{k=1}^{N}\left|l_{k}(\omega)\right|^{2} \\
& \sim \frac{\sigma_{v}^{2}}{2} \chi_{2 Q N}^{2}\left(\frac{\sum_{q=0}^{Q-1}\left\|\mathbf{H}\left(\omega_{q}\right) \mathbf{s}_{A}\left(\omega_{q}\right)\right\|^{2}}{\sigma_{v}^{2} / 2}\right) \\
& =\frac{\sigma_{v}^{2}}{2} \chi_{2 Q N}^{2}\left(\frac{\left\|\mathbf{H} \mathbf{s}_{A}\right\|^{2}}{\sigma_{v}^{2} / 2}\right) .
\end{aligned}
$$

Hence, we obtain the mean of $\Upsilon$ as follows:

$$
E\{\Upsilon\}=\sigma_{v}^{2} N Q+\left\|\mathbf{H} \mathbf{s}_{A}\right\|^{2}
$$

which yields the approximation in terms of the moments [36]

$$
E\left\{\frac{1}{\Upsilon}\right\}=\left(N Q \sigma_{v}^{2}+\left\|\mathbf{H} \mathbf{s}_{A}\right\|^{2}\right)^{-1} .
$$

To obtain a closed-form expression for $E\{(\Phi) /(\Upsilon)\}$, we approximate $E\{(\Phi) /(\Upsilon)\} \approx E\{\Phi\} E\{(1) /(\Upsilon)\}$. The approximation is reasonable when the correlation coefficient [37] of the random variables $\Phi$ and $\Upsilon^{-1}$ is small, since

$$
\rho=\frac{E\left\{\frac{\Phi}{\Upsilon}\right\}-E\{\Phi\} E\left\{\frac{1}{\Upsilon}\right\}}{\sigma_{\Phi} \sigma_{\Upsilon-1}}
$$

where $\sigma_{\Phi}$ and $\sigma_{\Upsilon-1}$ are the square roots of the second order central moments of $\Phi$ and $\Upsilon^{-1}$, respectively. A small $|\rho| \in$ $[0,1]$ means a good approximation. The bottom panel of Fig. 1 shows the value of $\rho$ versus SNR and $Q$ by simulation, which 
confirms that the approximation is valid. Next, plugging (103) and (93) into (90) yields

$$
\mathrm{SNRG}_{\mathrm{asym}} \approx \frac{\left\|\mathbf{s}_{A}\right\|^{2}\left(\left\|\mathbf{H}^{T} \mathbf{H}^{*} \mathbf{s}_{A}^{*}\right\|^{2}+\sigma_{v}^{2}\|\mathbf{H}\|^{2}\right)}{(P / N)\left\|\mathbf{H}^{T} \mathbf{s}_{B}\right\|^{2}\left(\left\|\mathbf{H} \mathbf{s}_{A}\right\|^{2}+\sigma_{v}^{2} N Q\right)}
$$

We comment on this expression of the gain $\mathrm{SNRG}_{\text {asym }}$ as follows.

1) If the time reversed signal is noise free, i.e., $\sigma_{v}^{2}=0$ in $\mathbf{y}_{m}$, this gain reduces to the maximal SNR gain (71) (see also [2] for the maximal SNR gain with a single antenna pair).

2) When $M_{Y}$ snapshots of $\mathbf{y}_{m}, m=1, \ldots, M_{Y}$ are used for averaging to reduce the noise variance in the time-reversed signal, this gain takes the form of (105) except that $\sigma_{v}^{2}$ is replaced by $\sigma_{v}^{2} / M_{Y}$

3) In our development, we assume that $\mathbf{H}$ is known. Thus, this gain represents the asymptotic theoretical performance gain of the TR GLRT (TR-GLRT) over the conventional GLRT detector, i.e., when the unknown $\mathbf{H}$ is estimated using an asymptotic large number of measurements.

\section{EXPERIMENTS AND PERFORMANCE RESUlTS}

In this section, we illustrate the performance of the detectors presented before with experimental data measured in a laboratory environment.

\section{A. Experimental Setup}

The detection experiment geometry is shown in Fig. 2. We illuminate the scattering medium with a broadband pulse of 2 $\mathrm{GHz}$, with center frequency at $5 \mathrm{GHz}$. This signal is generated by an Agilent 89610A vector signal analyzer. We capture and record the in-phase (I channel) and quadrature (Q channel) streams of the impulse response. The transmitter and receiver antennas are two horn antennas, both operating in the band 4 to $6 \mathrm{GHz}$. This $2-\mathrm{GHz}$ band is divided evenly into $Q=51$ bins. The two antenna arrays $A$ and $B$ are synthesized by sliding each horn antenna in their own slider $A$ or $B$, and stopping at one of ten predetermined locations separated by $10.16 \mathrm{~cm}$. The two sliders are aligned, with their two closest end points separated by $48.26 \mathrm{~cm}$. These ten locations in each slider lead to 100 possible transmit-receive pairs. Various combinations of these pairs produce arrays with different aperture sizes and number of antennas. To indicate which antenna locations are used in a given array, we number each location explicitly and refer to them within brackets. For example, the notation $B=[1,3,6]$ specifies that array $B$ is defined by the horn antenna in slider $B$ at positions 1, 3, and 6 (see the top panel of Fig. 2). The radiated signal is reflected by a number of scatterers placed in front of an absorber wall. A mix of dielectric pipes and copper rods creates the scattering environment. The target, surrounded by scatterers, is a single copper pipe with $1.5 \mathrm{~cm}$ in diameter and $2.5 \mathrm{~m}$ in length. The scatterers are dielectric pipes with $3 \mathrm{~cm}$ in diameter and $2.5 \mathrm{~m}$ in length. Besides the dielectric pipes, we add a few copper pipes as scattering objects. Two dielectric pipes are wrapped with aluminum foils to increase the reflectivity. All the pipes stand vertically. We measure the cylindrical wave propagating between the antennas and the scattering
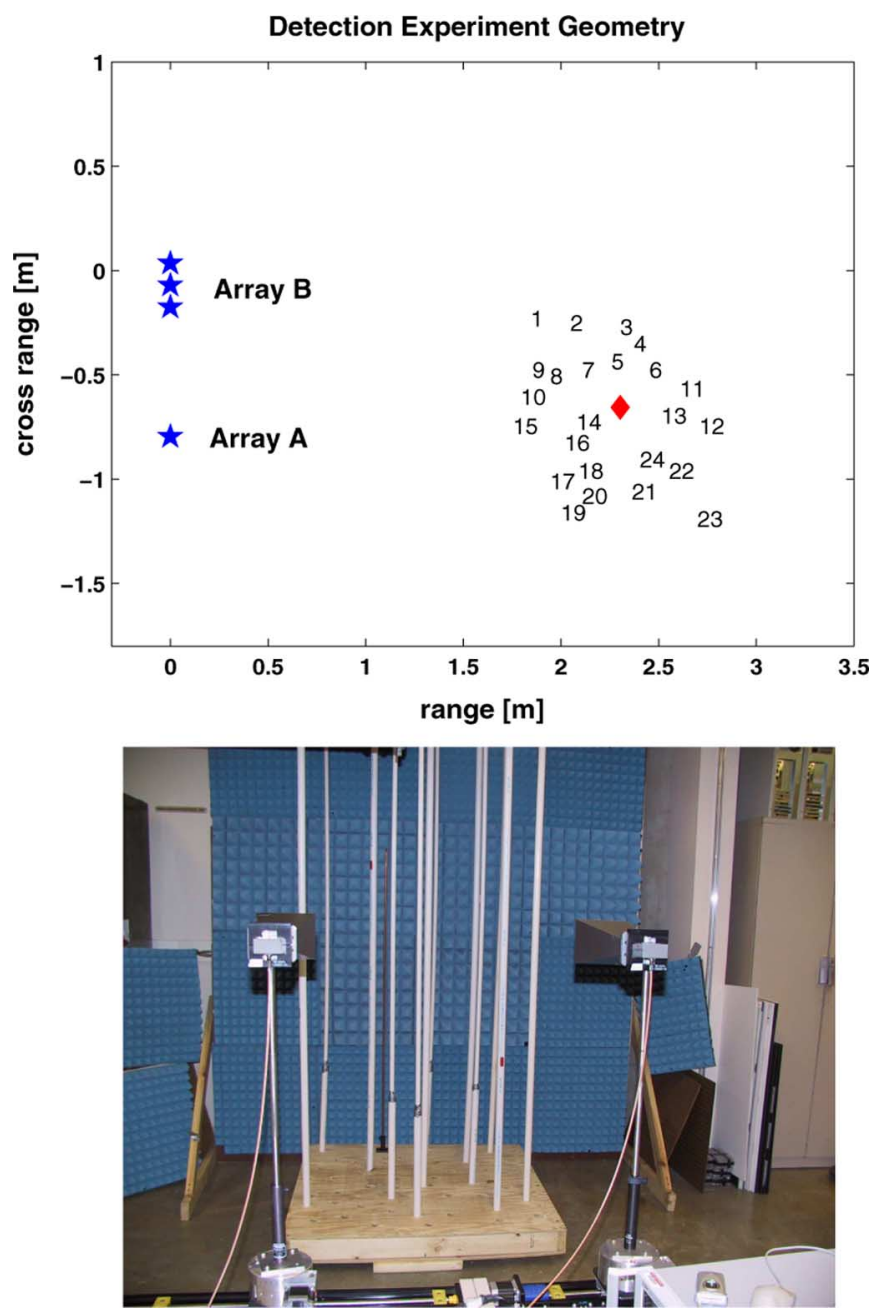

Fig. 2. Top panel: detection experiment geometry. The numbers indicate scatterers positions; the diamond indicates target location; stars indicate antenna positions. Bottom panel: photo of the laboratory. Dielectric rods are the scatterers; copper pipes are the targets. Two horn antennas, one transmits and one receives, synthesize two linear arrays, $\mathrm{A}$ and $\mathrm{B}$, by moving along two position sliders.

objects. During the measuring process, the two horn antennas point to the target area with size $1.2 \mathrm{~m} \times 1.2 \mathrm{~m}$ and centered at $\left(X_{c}, Y_{c}\right)=(2.3 \mathrm{~m},-0.65 \mathrm{~m})$.

To generate receiver operating characteristic (ROC) curves, we add artificial (numerically generated) noise to the real data measurements at different SNR levels. To be consistent with the commonly used definition of SNR in the radar literature (e.g., [38] and [39]), this paper defines the SNR as follows:

$$
\mathrm{SNR}=\frac{\left\|\mathbf{H} \mathbf{s}_{A}\right\|^{2}}{N \sigma_{v}^{2}} .
$$

This definition (106) is a scaled version of the SNR given in our previous paper in [2].

We take $\sigma_{v}^{2}=\sigma_{w}^{2} ; P_{\mathrm{FA}}=0.01$; and synthesize 5000 Monte Carlo runs to generate the ROC curves. In the ROC plots, the solid lines labeled with "ana" plot the analytical performance predicted by the study in Section IV, while the markers plot the performance obtained by averaging the Monte Carlo runs. The ROC plots can be extended straightforward to false alarm rates, for example, $10^{-6}$ or smaller. 

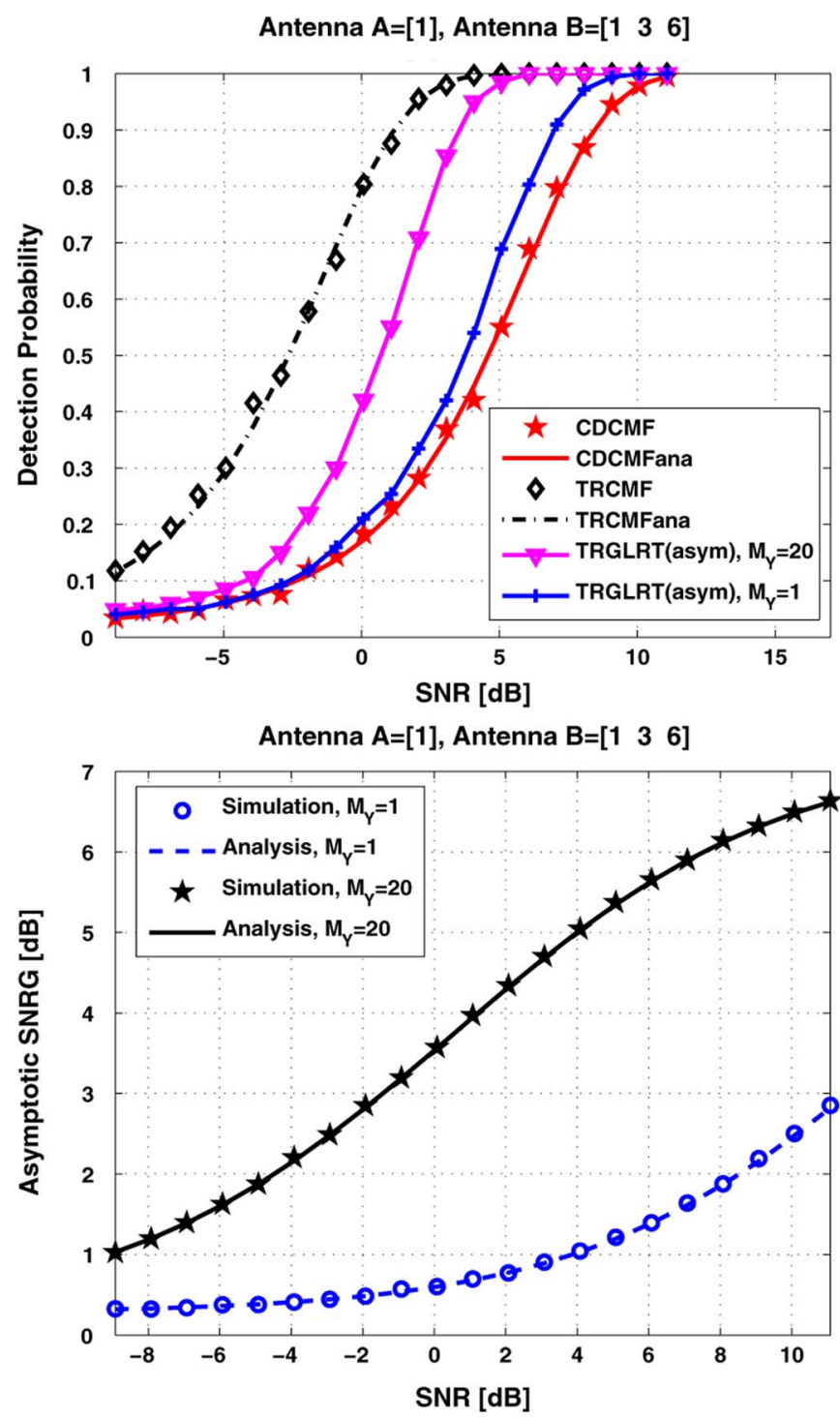

Fig. 3. SNR gain plot with 30 scatterers. Top panel: TRCMF, CDCMF and TR-GLRT(asym) versus SNR. The maximal SNR gain is $7.0 \mathrm{~dB}$ for TRCMF compared with CDCMF. The TR-GLRT(asym) has about a 3-dB gain over CDCMF. $P_{\mathrm{FA}}=0.01$. Bottom panel: Asymptotic SNR gain of TR-GLRT(asym) versus CDCMF.

\section{B. TR Detection Performance}

Fig. 3 depicts the ROC curves for the TRCMF, CDCMF, and TR-GLRT (asym) $_{\text {detectors, where the TR-GLRT }}$ (asym) detector is the TR-GLRT given in (41), except that the target channel is assumed known, i.e., estimated with an asymptotically large data set so that the estimated error is zero. The arrays are $A=[1]$ and $B=[1,3,6]$, i.e., have a single antenna and three antennas, respectively. There are 30 scatterers. The top panel in Fig. 3 shows that the theoretical performance (solid lines) match well the Monte Carlo trial results (markers) for both the TRCMF and CDCMF detectors. The maximal SNR gain of the TRCMF detector over the CDCMF detector in the study of Fig. 3 is $\mathrm{SNRG}_{\max }=7.0 \mathrm{~dB}$. The panel also displays the performance for two plots of the TR-GLRT (asym) $_{\text {detector, when }}$ $M_{Y}=1$ and $M_{Y}=20$ snapshots are available to reduce the noise component $\mathbf{v}$ by averaging before TR and retransmission. The bottom panel of Fig. 3 shows the asymptotic SNR gain
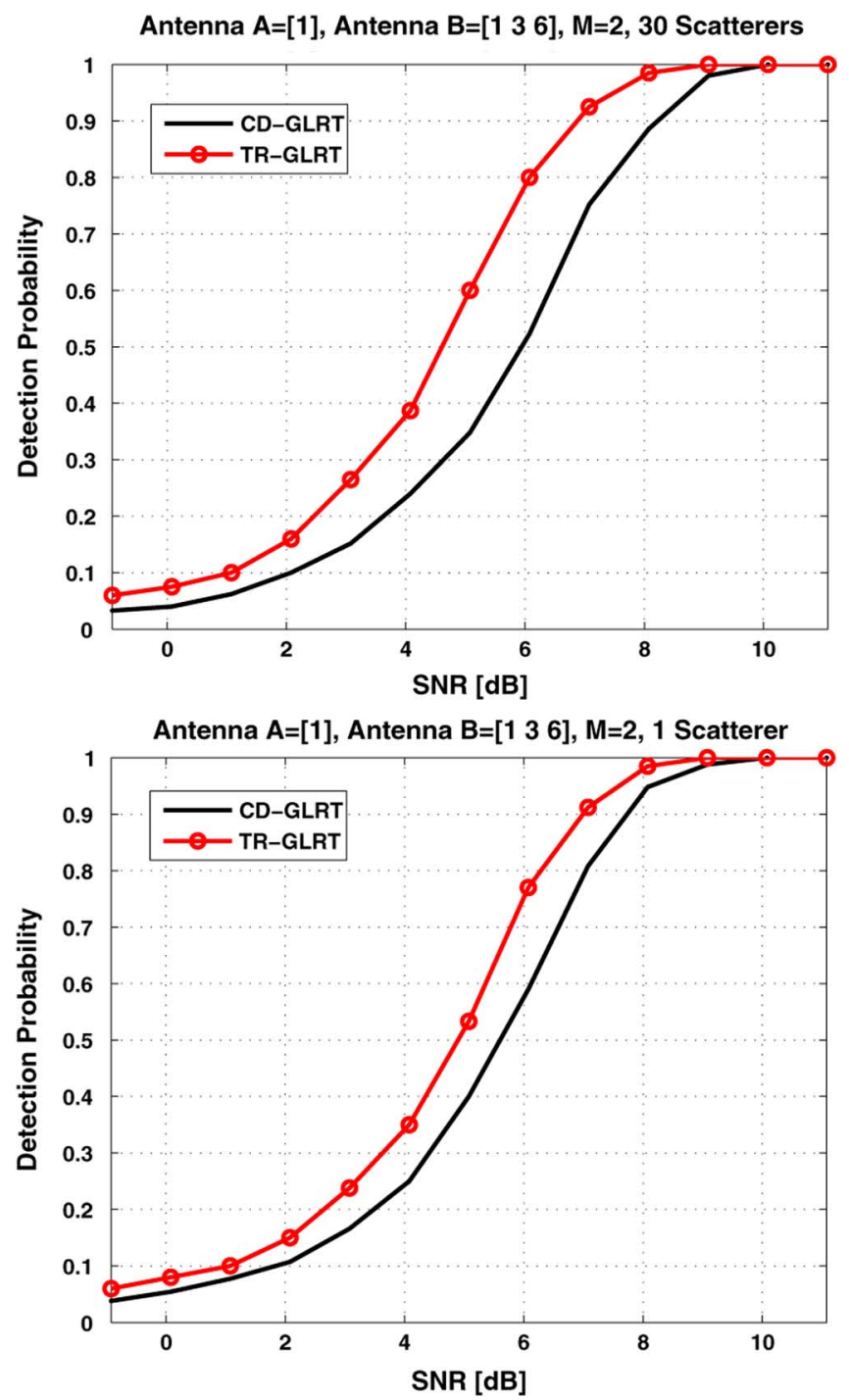

Fig. 4. Comparison of the ROCs for the TR-GLRT and the CD-GLRT detectors. The array configurations are $A=[1]$ and $B=[1,3,6]$. Number of snapshots $M=2 . P_{\mathrm{FA}}=0.01$. Top panel: 30 scatterers, SNR gain at $P_{D}=0.8$ is $1.8 \mathrm{~dB}$. Bottom panel: 1 scatterer, SNR gain at $P_{D}=0.8$ is $0.8 \mathrm{~dB}$. Richer scattering yields a larger SNR gain. Compared with Fig. 3, the TR gain is reduced.

(105) of the TR-GLRT $($ asym) detector over the CDCMF detector. The predicted $\mathrm{SNRG}_{\mathrm{asym}}$ (solid lines) matches well with the simulation results (markers). For example, with $M_{Y}=1$, we calculate, using the ROC curves in the top panel, a $1.5-\mathrm{dB}$ gain of the TR-GLRT (asym) detector over the CDCMF detector at $\mathrm{SNR}=7 \mathrm{~dB}$. The predicted $\mathrm{SNR}$ gain $\mathrm{SNRG}_{\mathrm{asym}}$ is read, from the bottom panel in Fig. 3, to be $1.7 \mathrm{~dB}$ at the same SNR level. When we use $M_{Y}=20$ snapshots, the asymptotic SNR gain is improved significantly. In this case, the variance of the noise in the clutter response channel estimate is reduced by a factor of 20, i.e., $\mathrm{SNR}=3 \mathrm{~dB}$. As observed from the bottom panel, the asymptotic SNR gain is now $4.5 \mathrm{~dB}$, a $2.8-\mathrm{dB}$ improvement over the $M_{Y}=1$ case. This indicates that the noise component in the time-reversed signal has a significant impact on the detection performance: a rich scattering but quiet environment is preferred for TR.

Fig. 4 displays the ROC curves for the TR-GLRT and the CD-GLRT detectors using $M=2$ snapshots, when the target is surrounded by 30 scatterers (top panel) and a single scatterer 

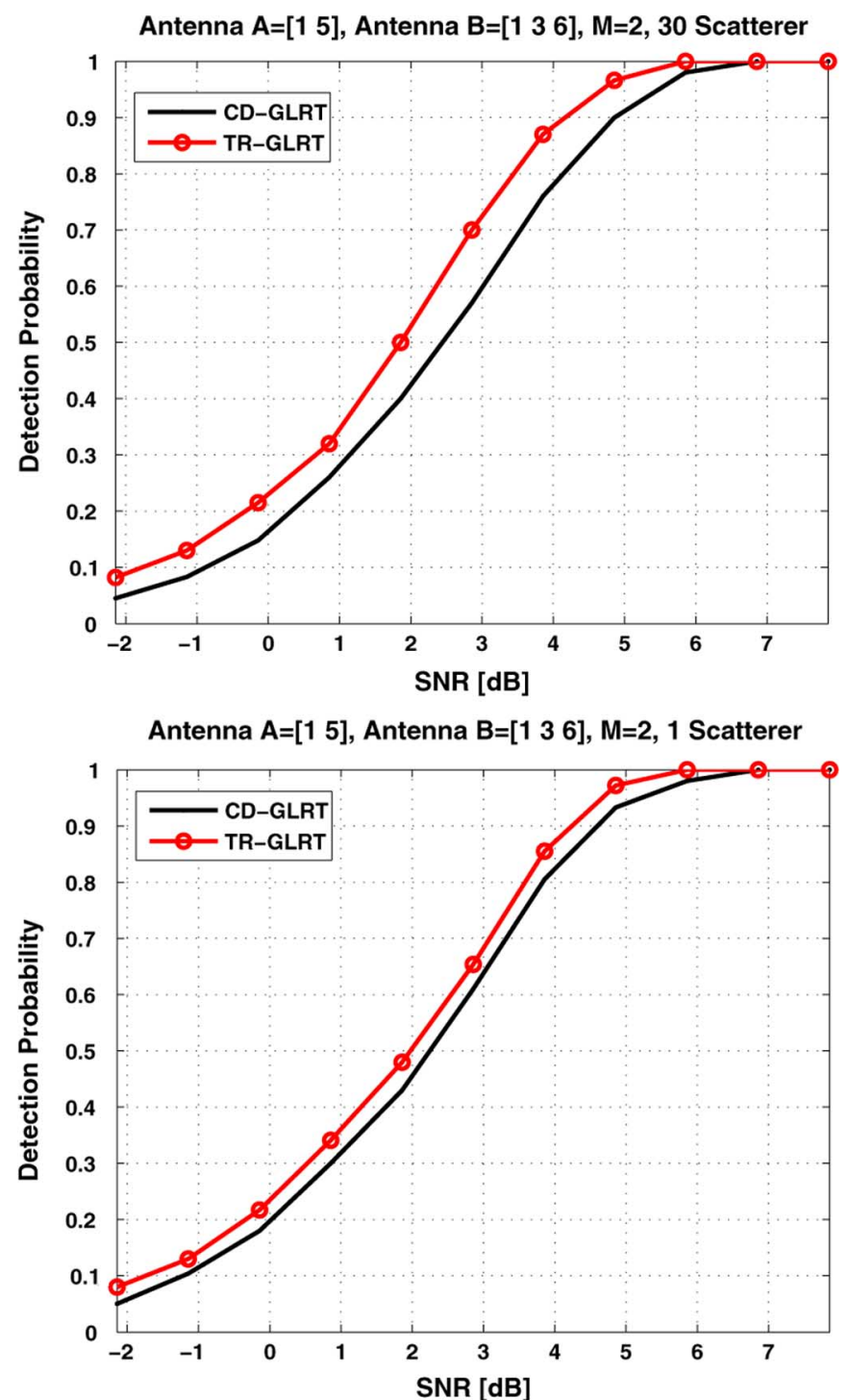

Fig. 5. Comparison of the ROCs for the TR-GLRT and the CD-GLRT detectors. The array configurations are $A=[1,5], B=[1,3,6]$. Number of snapshots $M=2$. Top panel: 30 scatterers, SNR gain at $P_{D}=0.8$ is $0.7 \mathrm{~dB}$. Bottom panel: 1 scatterer, SNR gain at $P_{D}=0.8$ is $0.2 \mathrm{~dB}$. Richer scattering yields a larger SNR gain.

(bottom panel). The top panel shows that the TR-GLRT detector has a gain of $1.6 \mathrm{~dB}$ over the CD-GLRT detector. This gain is reduced to $0.5 \mathrm{~dB}$ in the much less rich scattering environment of the bottom panel. The results also confirm that the analytical asymptotic expression $\mathrm{SNRG}_{\mathrm{asym}}$ is a good predictor of the actual SNR gain of the TR-GLRT detector over the CD-GLRT detector.

Fig. 5 considers a more general case, when an additional antenna is added. Now, the array $A$ has two antennas $A=[1,5]$, where antenna 5 is about $40 \mathrm{~cm}$ away from antenna 1 . The array $B$ has the same three antennas $B=[1,3,6]$ as in the previous two studies. The number of scatterers is 30 on the top panel and 1 on the bottom panel. Note that, to compare with the scenario with a single transmit antenna shown in Fig. 4, we normalize the SNR in Fig. 5 relative to the SNR in Fig. 4. Adding an additional antenna results in about a 3.2-dB increase in received signal power (channel gain.) The ROC curves in Fig. 5 indicate that the TR-GLRT detector gain over the CD-GLRT is reduced from $1.6 \mathrm{~dB}$ in Fig. 4 to $0.7 \mathrm{~dB}$ in Fig. 5 (top panels) and from 0.5 to $0.2 \mathrm{~dB}$ (bottom panels). This shows that the spatial diversity afforded by the larger array $A$ in this study, when compared to the case studied in Fig. 4, reduces the impact of the TR gain.

\section{TR for Synthetic Aperture Radar Imaging}

SARs provide a crucial technology for target detection and localization [40]-[42]. However, conventional SAR systems are not designed for imaging targets in a rich scattering environment. In this section, we examine the performance of TR when used in conjunction with SAR imaging when the target is concealed in clutter. We proposed TR SAR (TR-SAR) in [43] and [44]. To simplify the analysis, we interpret SAR imaging as beamforming, i.e., the conventional SAR data-collection and image formation process is a simple beamformer with sidelobe control [45], [46]. To demonstrate the advantage of TR-SAR over conventional SAR, we consider a simplified imaging scenario with the following assumptions: 1) The clutter is static and can be subtracted out to obtain the difference signals (i.e., conventional change detection). 2) We adopt a bi-static configuration (see, e.g., [47] and [48] for discussion of bi-static SAR) shown in Fig. 2 where array $\mathrm{B}$ has $N=10$ antennas and array A has one antenna. In other words, the single antenna in array A remains fixed, while the antenna in array B moves to ten different positions to synthesize array B. 3) The channel response is decomposed as

$$
h_{n}\left(\omega_{q}\right)=\zeta \bar{h}_{n}\left(\omega_{q}\right) e^{-j \omega_{q} \tau_{n}}, \quad n=1, \ldots, N
$$

where $\tau_{n}$ is the time delay of the direct path from the transmitter to the target, and to the receiver; the relative multipath channel is

$$
\bar{h}_{n}\left(\omega_{q}\right)=1+\Delta \bar{h}_{n}\left(\omega_{q}\right)
$$

where the multipath term is

$$
\Delta \bar{h}_{n}\left(\omega_{q}\right)=\sum_{l=1}^{L-1} \alpha_{l} e^{-j \Delta \tau_{l, n}}
$$

Symbols $\alpha_{l}, \tau_{l, n}$ denote the amplitude and differential delay of the $l$ th multipath; $\zeta$ denotes the target radar cross section (RCS). 4) For conventional SAR, we assume that the probe signal $S\left(\omega_{q}\right)=1$ (the equivalent time-domain signal is a sinc function and can be obtained by the inverse Fourier transform). For TR, we use the probe signal

$$
k_{p} S^{*}\left(\omega_{q}\right) \bar{h}_{n}^{*}\left(\omega_{q}\right)=k_{p} \bar{h}_{n}^{*}\left(\omega_{q}\right)
$$

where the normalization factor

$$
k_{p}=\sqrt{\frac{N \sum_{q=0}^{Q-1}\left|S\left(\omega_{q}\right)\right|^{2}}{\sum_{q=0}^{Q-1} \sum_{n=1}^{N}\left|\bar{h}_{n}\left(\omega_{q}\right) S\left(\omega_{q}\right)\right|^{2}}} .
$$

Hence, the received SAR data (for conventional change detection) can be written as

$$
P_{\mathrm{CD}}\left(\omega_{q}, B_{n}\right)=\zeta S\left(\omega_{q}\right) \bar{h}_{n}\left(\omega_{q}\right) e^{-j \omega_{q} \tau_{n}}+W\left(\omega_{q}, B_{n}\right)
$$

where $B_{n}$ is the $n$th antenna, $W\left(\omega_{q}, B_{n}\right) \sim \mathcal{C N}\left(0, \sigma_{w}^{2}\right)$ is the additive noise. To form an SAR image, we first stack $P_{\mathrm{CD}}\left(\omega_{q}, B_{n}\right)$ as a vector

$$
\mathbf{p}_{\mathrm{CD}}=\operatorname{vec}\left\{P_{\mathrm{CD}}\left(\omega_{q}, B_{n}\right)\right\} \text {. }
$$

The weighting coefficients for each pixel $\mathbf{x}$ in the image are given by $V_{q, n}(\mathbf{x})$ (for example, a windowed fast 
Fourier transform, or FFT [46]) and written as a vector $\mathbf{v}(\mathbf{x})=\operatorname{vec}\left\{V_{q, n}(\mathbf{x})\right\}$. We should note that the weighting coefficients $V_{q, n}(\mathbf{x})$ are two-dimensional in fast-time frequency (or range) domain and slow-time frequency (or Doppler) domain. Here we use a linear processing for SAR image reconstruction and detection, which is an approximation of the wavefront reconstruction method discussed in many SAR literature (e.g., [40]). Hence, the target radar cross section can be obtained by

$$
\begin{aligned}
\left|\hat{\zeta}_{\mathrm{CD}}\right|^{2}= & \left|\left\langle\mathbf{p}_{\mathrm{CD}}, \mathbf{v}(\mathbf{x})\right\rangle\right|^{2} \\
= & \mid \sum_{q=0}^{Q-1} \zeta \sum_{n=1}^{N} \bar{h}_{n}\left(\omega_{q}\right) e^{-j \omega_{q} \tau_{n}} V_{q, n}(\mathbf{x}) \\
& +\left.\sum_{q=0}^{Q-1} \sum_{n=1}^{N} W\left(\omega_{q}, A_{n}\right) V_{q, n}(\mathbf{x})\right|^{2} \\
= & \mid \sum_{q=0}^{Q-1} \zeta \sum_{n=1}^{N} e^{-j \omega_{q} \tau_{n}} V_{q, n}(\mathbf{x}) \\
& +\sum_{q=0}^{Q-1} \zeta \sum_{n=1}^{N} \Delta \bar{h}_{n}\left(\omega_{q}\right) e^{-j \omega_{q} \tau_{n}} V_{q, n}(\mathbf{x}) \\
& +\left.\sum_{q=0}^{Q-1} \sum_{n=1}^{N} W\left(\omega_{q}, A_{n}\right) V_{q, n}(\mathbf{x})\right|^{2} .
\end{aligned}
$$

In (115), the first term is the target phase history data; the second is induced by multipath that produces ghost images [43], [44]; the last term is the additive noise. We should note that in the second term of (115), the quantity $\Delta \bar{h}_{n}\left(\omega_{q}\right)$ is a complex number that creates a phase shift in addition to the target signature phase $e^{-j \omega_{q} \tau_{n}}$. It is this phase shift that induces the ghost images in conventional SAR processing. Time reversal removes the phase shift and produces a focused target image.

Using TR, the received SAR data is

$$
P_{\mathrm{TR}}\left(\omega_{q}, A_{n}\right)=\zeta S^{*}\left(\omega_{q}\right)\left|\bar{h}_{n 1}^{*}\left(\omega_{q}\right)\right|^{2} e^{-j \omega_{q} \tau_{n 1}}+W\left(\omega_{q}, A_{n}\right) .
$$

The vectorized SAR data and the target RCS are

$$
\mathbf{p}_{\mathrm{TR}}=\operatorname{vec}\left\{P_{\mathrm{TR}}\left(\omega_{q}, A_{n}\right)\right\}
$$

and

$$
\begin{aligned}
\left|\hat{\zeta}_{\mathrm{TR}}\right|^{2}= & \left|\left\langle\mathbf{p}_{\mathrm{TR}}, \mathbf{v}(\mathbf{x})\right\rangle\right|^{2} \\
= & \left.\left|\sum_{q=0}^{Q-1} \zeta k_{p} \sum_{n=1}^{N}\right| \bar{h}_{n}\left(\omega_{q}\right)\right|^{2} e^{-j \omega_{q} \tau_{n}} V_{q, n}(\mathbf{x}) \\
& +\left.\sum_{q=0}^{Q-1} \sum_{n=1}^{N} W\left(\omega_{q}, B_{n}\right) V_{q, n}(\mathbf{x})\right|^{2} .
\end{aligned}
$$

In (118), the first term is the focused target phase history data and the second term is the additive noise. We should note that the quantity $\left|\bar{h}_{n}\left(\omega_{q}\right)\right|^{2}$ in the first term of (118) is a real number that does not induce phase disturbances on the target signature phase $e^{-j \omega_{q} \tau_{n}}$. This is different from the first term in (114) where $\bar{h}_{n}\left(\omega_{q}\right)$ is a complex number due to the superposition of the multipath and the direct path. Compared with (115), the ghost images are removed in (118). As a result, the TR SAR

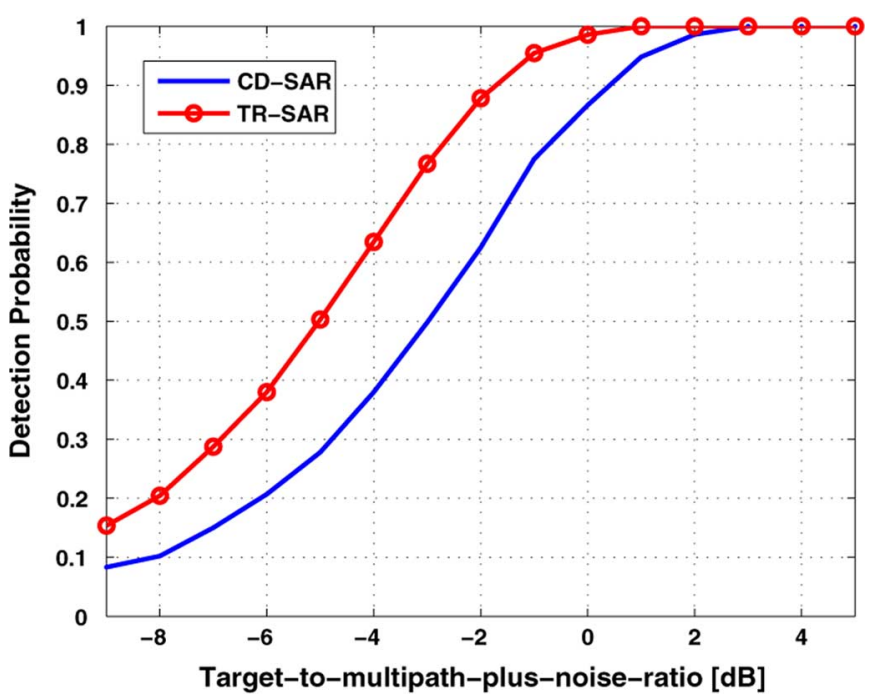

Fig. 6. TR detection versus conventional detection in SAR. $P_{\mathrm{fa}}=0.01$. The imaging geometry is shown in Fig. 2. The antenna collects SAR data at ten positions.

images produce a clear map of the target with improved resolution and detectability. We now compare the performance of TR-SAR with conventional SAR for detection. The imaging geometry is shown in Fig. 2. The experimental setup is described in Section VII-A. In Fig. 6, we compare the receiver operating characteristics based on (115) and (118). We choose the weighting coefficients $V_{q, n}(\mathbf{x})=e^{-j \omega_{q} \tau_{n}}$, i.e., the beamformer matches with the target response. We define the target-to-multipath-plus-noise ratio (TMNR) as

$$
\mathrm{TMNR}=\frac{\zeta^{2} Q^{2} N^{2}}{\zeta^{2}\left|\sum_{n=1}^{N} \sum_{q=0}^{Q-1} \Delta \bar{h}_{n}\left(\omega_{q}\right)\right|^{2} / Q+N \sigma_{w}^{2}} .
$$

Fig. 6 shows that TR-SAR has $2 \mathrm{~dB}$ gain over CD-SAR for the scattering environment in Fig. 2. This is significant in applications.

\section{CONCLUSION}

This paper derives the TR generalized likelihood ratio detector using antenna arrays. It studies analytically the performance of the TR detector and provides experimental validation with electromagnetic data. We showed that TR is an adaptive waveform transmission strategy. To analyze the impact of the noise contained in the time-reversed signal, we derived closed form expressions for the asymptotic SNR gain of the TR detector over the conventional detector, in the asymptotic limit of large data sets, when the number of snapshots available to estimate the target channel response goes to infinity.

In our analysis, we assume that the clutter response can be estimated and subtracted from the measurement. Under this assumption, the performance of TR is determined by noise and the target channel response. In a rich scattering environment, the target channel response shows a widely fluctuating frequency spectrum. This contrasts with the direct line-of-sight environment where the point target has a flat frequency spectrum. TR transmission allocates transmission power in the frequency range where the target response is strong, which improves the transmission efficiency. 
The TR SNR gain varies depending on the array configuration. To characterize the TR gain over the conventional method, we show that, for a multipath rich Rayleigh channel model and using one transmit antenna and two receiving antennas, the TR detector has a 4.7-dB gain over the conventional detector. Under ideal conditions, a two-antenna TR mirror array attains transmit array gain of $4.7 \mathrm{~dB}$, greater than the nominal array gain of $3 \mathrm{~dB}$. This gain results from the multipath diversity due to rich multipath scattering and the spatial diversity due to the multielement array with independent channel coefficients. For a multistatic configuration with $P$ antennas in the transmit array $A$ and $N$ antennas in the receive array $B$, we recommend to use an $N>P$ configuration to implement TR. We show, by analysis and experiments, that the TRM yields significant detection gain over conventional detection. A more comprehensive study of the impact of the array configuration on TR detection, including the array aperture and the array interelement spacing will be reported in a future paper.

\section{APPENDIX I}

\section{DERIVATION OF TR-GLRT DETECTOR}

We use (18) as the $m$ th test data snapshot. Under the alternative hypothesis for detection problem (40), this snapshot is given by

$$
\mathbf{z}_{m}=\left[\begin{array}{c}
\mathbf{H}^{*} \mathbf{s}_{A}^{*} \\
k_{m} \mathbf{H}^{T} \mathbf{H}^{*} \mathbf{s}_{A}^{*}
\end{array}\right]+\left[\begin{array}{c}
\mathbf{v}_{m}^{*} \\
k_{m} \mathbf{H}^{T} \mathbf{v}_{m}^{*}+\mathbf{w}_{m}
\end{array}\right]
$$

Let $\Omega(q)$ denote the covariance matrix of $\mathbf{z}_{m}\left(\omega_{q}\right)=$ $\left[\mathbf{y}_{m}^{* T}\left(\omega_{q}\right), \mathbf{x}_{m}^{T}\left(\omega_{q}\right)\right]^{T}$, i.e.,

$$
\boldsymbol{\Omega}(q)=\left[\begin{array}{cc}
\sigma_{v}^{2} \mathbf{I}_{N} & k_{m} \mathbf{H}^{*}\left(\omega_{q}\right) \sigma_{v}^{2} \\
k_{m} \mathbf{H}^{T}\left(\omega_{q}\right) \sigma_{v}^{2} & k_{m}^{2} \mathbf{H}^{T}\left(\omega_{q}\right) \mathbf{H}^{*}\left(\omega_{q}\right) \sigma_{v}^{2}+\sigma_{w}^{2} \mathbf{I}_{P}
\end{array}\right] .
$$

Using the matrix inversion lemma, and the block matrix inversion lemma, [30], we obtain that

$$
\boldsymbol{\Omega}^{-1}(q)=\left[\begin{array}{cc}
\frac{1}{\sigma_{v}^{2}} \mathbf{I}_{N}+\frac{k_{m}^{2}}{\sigma_{w}^{2}} \mathbf{H}^{*}\left(\omega_{q}\right) \mathbf{H}^{T}\left(\omega_{q}\right) & -\frac{k_{m}}{\sigma_{w}^{2}} \mathbf{H}^{*}\left(\omega_{q}\right) \\
-\frac{k_{m}}{\sigma_{w}^{2}} \mathbf{H}^{T}\left(\omega_{q}\right) & \frac{1}{\sigma_{w}^{2}} \mathbf{I}_{P}
\end{array}\right] .
$$

Applying the identity for determinants of block matrices

$$
\operatorname{det}\left(\left[\begin{array}{ll}
\mathbf{A} & \mathbf{B} \\
\mathbf{C} & \mathbf{D}
\end{array}\right]\right)=\operatorname{det}(\mathbf{A}) \operatorname{det}\left(\mathbf{D}-\mathbf{C A}^{-1} \mathbf{B}\right)
$$

to (121) yields

$$
|\boldsymbol{\Omega}(q)|=\left|\sigma_{v}^{2} \mathbf{I}_{N}\right|\left|\sigma_{w}^{2} \mathbf{I}_{P}\right|=\left(\sigma_{v}^{2}\right)^{N}\left(\sigma_{w}^{2}\right)^{P} .
$$

The pdfs $p_{1}(\mathbf{Z}, \mathbf{H})$ and $p_{0}(\mathbf{Z})$ conditioned on the power normalization factors $k_{m}$ under hypotheses $\mathbb{H}_{1}$ and $\mathfrak{H}_{0}$, respectively, are given by (125)-(128), shown at the bottom of the page. Adopting the GLR principle by taking the logarithm of the

$$
\begin{aligned}
& p_{1}(\mathbf{Z}, \mathbf{H})=\prod_{q=0}^{Q-1} \frac{1}{\pi \operatorname{det}(\boldsymbol{\Omega}(q))} \exp \left\{-\sum_{m=1}^{M}\left[\begin{array}{l}
\mathbf{y}_{m}^{*}\left(\omega_{q}\right)-\mathbf{H}^{*}\left(\omega_{q}\right) \mathbf{s}_{A}^{*}\left(\omega_{q}\right) \\
\mathbf{x}_{m}\left(\omega_{q}\right)-k_{m} \mathbf{H}^{T}\left(\omega_{q}\right) \mathbf{H}^{*}\left(\omega_{q}\right) \mathbf{s}_{A}^{*}\left(\omega_{q}\right)
\end{array}\right]^{H} \boldsymbol{\Omega}^{-1}(q)\right. \\
& \left.\left[\begin{array}{c}
\mathbf{y}_{m}^{*}\left(\omega_{q}\right)-\mathbf{H}^{*}\left(\omega_{q}\right) \mathbf{s}_{A}^{*}\left(\omega_{q}\right) \\
\mathbf{x}_{m}\left(\omega_{q}\right)-k_{m} \mathbf{H}^{T}\left(\omega_{q}\right) \mathbf{H}^{*}\left(\omega_{q}\right) \mathbf{s}_{A}^{*}\left(\omega_{q}\right)
\end{array}\right]\right\} \\
& =\prod_{q=0}^{Q-1} \frac{1}{\pi\left(\sigma_{w}^{2}\right)^{P}\left(\sigma_{v}^{2}\right)^{N}} \exp \left\{\sum_{m=1}^{M}-\left(\mathbf{y}_{m}^{*}\left(\omega_{q}\right)-\mathbf{H}^{*}\left(\omega_{q}\right) \mathbf{s}_{A}^{*}\left(\omega_{q}\right)\right)^{H}\left[\frac{1}{\sigma_{v}^{2}} \mathbf{I}_{N}\right.\right. \\
& \left.+\frac{k_{m}^{2}}{\sigma_{w}^{2}} \mathbf{H}^{*}\left(\omega_{q}\right) \mathbf{H}^{T}\left(\omega_{q}\right)\right]\left(\mathbf{y}_{m}^{*}\left(\omega_{q}\right)-\mathbf{H}^{*}\left(\omega_{q}\right) \mathbf{s}_{A}^{*}\left(\omega_{q}\right)\right) \\
& +2 \Re\left\{\frac{k_{m}}{\sigma_{w}^{2}}\left(\mathbf{x}_{m}\left(\omega_{q}\right)-k_{m} \mathbf{H}^{T}\left(\omega_{q}\right) \mathbf{H}^{*}\left(\omega_{q}\right) \mathbf{s}_{A}^{*}\left(\omega_{q}\right)\right)^{H}\right. \\
& \left.\times \mathbf{H}^{T}\left(\omega_{q}\right)\left(\mathbf{y}_{m}^{*}\left(\omega_{q}\right)-\mathbf{H}^{*}\left(\omega_{q}\right) \mathbf{s}_{A}^{*}\left(\omega_{q}\right)\right)\right\} \\
& \left.-\frac{1}{\sigma_{w}^{2}}\left\|\mathbf{x}_{m}\left(\omega_{q}\right)-k_{m} \mathbf{H}^{T}\left(\omega_{q}\right) \mathbf{H}^{*}\left(\omega_{q}\right) \mathbf{s}_{A}^{*}\left(\omega_{q}\right)\right\|^{2}\right\} \\
& =\prod_{q=0}^{Q-1} \frac{1}{\pi\left(\sigma_{w}^{2}\right)^{P}\left(\sigma_{v}^{2}\right)^{N}} \exp \left\{-\sum_{m=1}^{M} \frac{\left\|\mathbf{x}_{m}\left(\omega_{q}\right)\right\|^{2}}{\sigma_{w}^{2}}-\frac{\left\|\mathbf{y}_{m}\left(\omega_{q}\right)\right\|^{2}}{\sigma_{v}^{2}}\right. \\
& +2 \Re\left\{\frac{k_{m}}{\sigma_{w}^{2}} \mathbf{y}_{m}^{T}\left(\omega_{q}\right) \mathbf{H}^{*}\left(\omega_{q}\right) \mathbf{x}_{m}\left(\omega_{q}\right)+\frac{1}{\sigma_{v}^{2}} \mathbf{y}_{m}^{T}\left(\omega_{q}\right) \mathbf{H}^{*}\left(\omega_{q}\right) \mathbf{s}_{A}^{*}\left(\omega_{q}\right)\right\} \\
& \left.-\frac{1}{\sigma_{v}^{2}}\left\|\mathbf{H}^{*}\left(\omega_{q}\right) \mathbf{s}_{A}^{*}\left(\omega_{q}\right)\right\|^{2}-\frac{k_{m}^{2}}{\sigma_{w}^{2}}\left\|\mathbf{H}^{T}\left(\omega_{q}\right) \mathbf{y}_{m}^{*}\left(\omega_{q}\right)\right\|^{2}\right\} \\
& p_{0}(\mathbf{Z})=\prod_{q=0}^{Q-1} \frac{1}{\pi\left(\sigma_{w}^{2}\right)^{P}\left(\sigma_{v}^{2}\right)^{N}} \exp \left\{-\sum_{m=1}^{M} \frac{\left\|\mathbf{x}_{m}\left(\omega_{q}\right)\right\|^{2}}{\sigma_{w}^{2}}\right\} \exp \left\{-\sum_{m=1}^{M} \frac{\left\|\mathbf{y}_{m}\left(\omega_{q}\right)\right\|^{2}}{\sigma_{v}^{2}}\right\} .
\end{aligned}
$$


ratio of the two pdfs (127) and (128) evaluated at the maximumlikelihood estimate of $\mathbf{H}$ yields the following test statistic:

$$
\begin{aligned}
\ell_{\mathrm{TR}-\mathrm{GLRT}}(\mathbf{Z})= & \ln p_{1}(\mathbf{Z})-\ln p_{0}(\mathbf{Z}) \\
= & \sum_{m=1}^{M} \sum_{q=0}^{Q-1}\left\{2 \mathfrak { R } \left\{\frac{k_{m}}{\sigma_{w}^{2}} \mathbf{y}_{m}^{T}\left(\omega_{q}\right) \widehat{\mathbf{H}}^{*}\left(\omega_{q}\right) \mathbf{x}_{m}\left(\omega_{q}\right)\right.\right. \\
& \left.+\frac{1}{\sigma_{v}^{2}} \mathbf{y}_{m}^{T}\left(\omega_{q}\right) \widehat{\mathbf{H}}^{*}\left(\omega_{q}\right) \mathbf{s}_{A}^{*}\left(\omega_{q}\right)\right\} \\
& -\frac{1}{\sigma_{v}^{2}}\left\|\widehat{\mathbf{H}}^{*}\left(\omega_{q}\right) \mathbf{s}_{A}^{*}\left(\omega_{q}\right)\right\|^{2} \\
& \left.-\frac{k_{m}^{2}}{\sigma_{w}^{2}}\left\|\widehat{\mathbf{H}}^{T}\left(\omega_{q}\right) \mathbf{y}_{m}^{*}\left(\omega_{q}\right)\right\|^{2}\right\} \\
= & \frac{1}{\sigma_{w}^{2} \sigma_{v}^{2}} \sum_{q=0}^{Q-1}\left\{2 \mathfrak{R}\left\{\operatorname{Tr}\left[\hat{\mathbf{H}}^{*}\left(\omega_{q}\right) \mathbf{A}\left(\omega_{q}\right)\right]\right\}\right. \\
& -\operatorname{Tr}\left[\hat{\mathbf{H}}^{T}\left(\omega_{q}\right) \widehat{\mathbf{H}}^{*}\left(\omega_{q}\right) \mathbf{C}\left(\omega_{q}\right)\right] \\
& \left.-\operatorname{Tr}\left[\hat{\mathbf{H}}^{*}\left(\omega_{q}\right) \widehat{\mathbf{H}}^{T}\left(\omega_{q}\right) \mathbf{D}\left(\omega_{q}\right)\right]\right\}
\end{aligned}
$$

where

$$
\begin{aligned}
\mathbf{A}\left(\omega_{q}\right)= & \sum_{m=1}^{M}\left[k_{m} \sigma_{v}^{2} \mathbf{x}_{m}\left(\omega_{q}\right) \mathbf{y}_{m}^{T}\left(\omega_{q}\right)\right. \\
& \left.+\sigma_{w}^{2} \mathbf{s}_{A}^{*}\left(\omega_{q}\right) \mathbf{y}_{m}^{T}\left(\omega_{q}\right)\right] \\
\mathbf{C}\left(\omega_{q}\right)= & M \sigma_{w}^{2} \mathbf{s}_{A}^{*}\left(\omega_{q}\right) \mathbf{s}_{A}^{T}\left(\omega_{q}\right) \\
\mathbf{D}\left(\omega_{q}\right)= & \sum_{m=1}^{M} k_{m}^{2} \sigma_{v}^{2} \mathbf{y}_{m}^{*}\left(\omega_{q}\right) \mathbf{y}_{m}^{T}\left(\omega_{q}\right)
\end{aligned}
$$

Next, we derive the maximum likelihood estimate $\hat{\mathbf{H}}\left(\omega_{q}\right)$ of $\mathbf{H}\left(\omega_{q}\right)$ under $\mathbb{H}_{1}$ that appears in (129). We neglect the dependency of $k_{m}$ on the target channel response and so $\hat{\mathbf{H}}\left(\omega_{q}\right)$ is only an approximation to the true ML estimate. The validity of such an assumption is shown in Appendix III. Taking the partial derivative of $\ln p_{1}(\mathbf{z} ; \mathbf{H})$ with respect to $\mathbf{H}^{*}\left(\omega_{q}\right)$, and ignoring the constant terms, yields

$$
\begin{aligned}
\frac{\partial[\ln }{\left.\partial \mathbf{H}_{1}(\mathbf{Z} ; \mathbf{H})\right]} & \\
= & \frac{\partial}{\partial \mathbf{H}_{q}^{*}\left(\omega_{q}\right)} \sum_{m=1}^{M} \sum_{q=0}^{Q-1}\left\{-\frac{\left\|\mathbf{x}_{m}\left(\omega_{q}\right)\right\|^{2}}{\sigma_{w}^{2}}\right. \\
& -\frac{\left\|\mathbf{y}_{m}\left(\omega_{q}\right)\right\|^{2}}{\sigma_{v}^{2}} \\
& +2 \mathfrak{R}\left\{\frac{k_{m}}{\sigma_{w}^{2}} \mathbf{y}_{m}^{T}\left(\omega_{q}\right) \mathbf{H}^{*}\left(\omega_{q}\right) \mathbf{x}_{m}\left(\omega_{q}\right)\right. \\
& \left.+\frac{1}{\sigma_{v}^{2}} \mathbf{y}_{m}^{T}\left(\omega_{q}\right) \mathbf{H}^{*}\left(\omega_{q}\right) \mathbf{s}_{A}^{*}\left(\omega_{q}\right)\right\} \\
& -\frac{1}{\sigma_{v}^{2}}\left\|\mathbf{H}^{*}\left(\omega_{q}\right) \mathbf{s}_{A}^{*}\left(\omega_{q}\right)\right\|^{2} \\
& \left.-\frac{k_{m}^{2}}{\sigma_{w}^{2}}\left\|\mathbf{H}^{T}\left(\omega_{q}\right) \mathbf{y}_{m}^{*}\left(\omega_{q}\right)\right\|^{2}\right\}
\end{aligned}
$$

$$
\begin{aligned}
= & \frac{\partial}{\partial \mathbf{H}^{*}\left(\omega_{q}\right)} \sum_{q=0}^{Q-1}\left\{-\sum_{m=1}^{M} \frac{\left\|\mathbf{x}_{m}\left(\omega_{q}\right)\right\|^{2}}{\sigma_{w}^{2}}\right. \\
& -\sum_{m=1}^{M} \frac{\left\|\mathbf{y}_{m}\left(\omega_{q}\right)\right\|^{2}}{\sigma_{v}^{2}} \\
& +2 \Re\left\{\operatorname{Tr}\left[\mathbf{H}^{*}\left(\omega_{q}\right) \mathbf{A}\left(\omega_{q}\right)\right]\right\} \\
& -\operatorname{Tr}\left[\mathbf{H}^{T}\left(\omega_{q}\right) \mathbf{H}^{*}\left(\omega_{q}\right) \mathbf{C}\left(\omega_{q}\right)\right] \\
& \left.-\operatorname{Tr}\left[\mathbf{H}^{*}\left(\omega_{q}\right) \mathbf{H}^{T}\left(\omega_{q}\right) \mathbf{D}\left(\omega_{q}\right)\right]\right\} .
\end{aligned}
$$

Utilizing the rule of treating complex variable $v$ and its conjugate $v^{*}$ as two distinct quantities, [49], i.e.,

$$
\frac{\partial|v|^{2}}{\partial v^{*}}=v
$$

we obtain the following expression that involves taking the derivative of a trace with respect to complex numbers

$\frac{\partial\left[\ln p_{1}(\mathbf{Z} ; \mathbf{H})\right]}{\partial \mathbf{H}^{*}\left(\omega_{q}\right)}=-\mathbf{C}\left(\omega_{q}\right) \mathbf{H}^{T}\left(\omega_{q}\right)-\mathbf{H}^{T}\left(\omega_{q}\right) \mathbf{D}\left(\omega_{q}\right)+\mathbf{A}\left(\omega_{q}\right)$.

Equating to zero yields the Lyapunov Sylvester matrix equation [50]

$$
\widehat{\mathbf{H}}\left(\omega_{q}\right) \mathbf{C}^{T}\left(\omega_{q}\right)+\mathbf{D}^{T}\left(\omega_{q}\right) \widehat{\mathbf{H}}\left(\omega_{q}\right)=\mathbf{A}^{T}\left(\omega_{q}\right) .
$$

Using Kronecker products and the vec operator, we get as follows:

$$
\begin{aligned}
\operatorname{vec}\left\{\mathbf{A}^{T}\left(\omega_{q}\right)\right\} & =\mathbf{G}\left(\omega_{q}\right) \operatorname{vec}\left\{\hat{\mathbf{H}}\left(\omega_{q}\right)\right\} \\
\mathbf{G}\left(\omega_{q}\right) & =\mathbf{C}\left(\omega_{q}\right) \otimes \mathbf{I}_{N}+\mathbf{I}_{P} \otimes \mathbf{D}^{T}\left(\omega_{q}\right)
\end{aligned}
$$

which leads to

$$
\operatorname{vec}\left\{\widehat{\mathbf{H}}\left(\omega_{q}\right)\right\}=\left[\mathbf{G}\left(\omega_{q}\right)\right]^{-1} \operatorname{vec}\left\{\mathbf{A}^{T}\left(\omega_{q}\right)\right\} .
$$

The ML estimate $\widehat{\mathbf{H}}\left(\omega_{q}\right)$ can be obtained by un-doing the vectorization of $\operatorname{vec}\left\{\hat{\mathbf{H}}\left(\omega_{q}\right)\right\}$ given in (140). However, caution should be taken when completing this ML estimate from (140) since it is often slow and ill-conditioned. Other standard solution methods for (137) are the Bartels-Stewart method, [51], and the Hessenberg-Schur method [52]. Finally, we obtain the channel estimate

$$
\widehat{\mathbf{H}}=\operatorname{diag}\left[\widehat{\mathbf{H}}\left(\omega_{0}\right), \ldots, \widehat{\mathbf{H}}\left(\omega_{Q-1}\right)\right] .
$$

\section{APPENDIX II}

DERIVATION OF THE CD-GLRT DETECTOR

Under the alternative hypothesis for the detection problem (42), the $m$ th test data is given by (26)

$$
\mathbf{u}_{m}=\left[\begin{array}{c}
\mathbf{H} \mathbf{s}_{A} \\
\beta \mathbf{H}^{T} \mathbf{s}_{B}
\end{array}\right]+\left[\begin{array}{c}
\mathbf{v}_{m} \\
\mathbf{w}_{m}
\end{array}\right], \forall m .
$$


The pdfs $p_{1}(\mathbf{U}, \mathbf{H})$ and $p_{0}(\mathbf{U})$, under hypotheses $\mathbb{H}_{1}$ and $\mathbb{H}_{0}$, respectively, are given by

$$
\begin{aligned}
p_{1}(\mathbf{U}, \mathbf{H}) & \\
= & \prod_{q=0}^{Q-1} \frac{1}{\pi\left(\sigma_{v}^{2}\right)^{N}\left(\sigma_{w}^{2}\right)^{P}} \\
& \times \exp \left\{-\sum_{m=1}^{M} \frac{1}{\sigma_{v}^{2}}\left\|\mathbf{y}_{m}\left(\omega_{q}\right)-\mathbf{H}\left(\omega_{q}\right) \mathbf{s}_{A}\left(\omega_{q}\right)\right\|^{2}\right\} \\
& \times \exp \left\{-\sum_{m=1}^{M} \frac{1}{\sigma_{w}^{2}}\left\|\mathbf{r}_{m}\left(\omega_{q}\right)-\beta \mathbf{H}^{T}\left(\omega_{q}\right) \mathbf{s}_{B}\left(\omega_{q}\right)\right\|^{2}\right\}
\end{aligned}
$$$$
=\prod_{q=0}^{Q-1} \frac{1}{\pi\left(\sigma_{w}^{2}\right)^{P}\left(\sigma_{v}^{2}\right)^{N}}
$$$$
\times \exp \left\{-\sum_{m=1}^{M} \frac{\left\|\mathbf{r}_{m}\left(\omega_{q}\right)\right\|^{2}}{\sigma_{w}^{2}}-\frac{\left\|\mathbf{y}_{m}\left(\omega_{q}\right)\right\|^{2}}{\sigma_{v}^{2}}\right.
$$$$
+2 \Re\left\{\frac{\beta}{\sigma_{w}^{2}} \mathbf{s}_{B}^{H}\left(\omega_{q}\right) \mathbf{H}^{*}\left(\omega_{q}\right) \mathbf{r}_{m}\left(\omega_{q}\right)\right.
$$$$
\left.+\frac{1}{\sigma_{v}^{2}} \mathbf{y}_{m}^{T}\left(\omega_{q}\right) \mathbf{H}^{*}\left(\omega_{q}\right) \mathbf{s}_{A}^{*}\left(\omega_{q}\right)\right\}
$$$$
-\frac{1}{\sigma_{v}^{2}}\left\|\mathbf{H}\left(\omega_{q}\right) \mathbf{s}_{A}\left(\omega_{q}\right)\right\|^{2}
$$$$
\left.-\frac{\beta^{2}}{\sigma_{w}^{2}}\left\|\mathbf{H}^{T}\left(\omega_{q}\right) \mathbf{s}_{B}\left(\omega_{q}\right)\right\|^{2}\right\}
$$

$p_{0}(\mathbf{U})$

$$
\begin{aligned}
= & \prod_{q=0}^{Q-1} \frac{1}{\pi\left(\sigma_{w}^{2}\right)^{P}\left(\sigma_{v}^{2}\right)^{N}} \\
& \times \exp \left\{-\sum_{m=1}^{M} \frac{\left\|\mathbf{r}_{m}\left(\omega_{q}\right)\right\|^{2}}{\sigma_{w}^{2}}\right\} \\
& \times \exp \left\{-\sum_{m=1}^{M} \frac{\left\|\mathbf{y}_{m}\left(\omega_{q}\right)\right\|^{2}}{\sigma_{v}^{2}}\right\} .
\end{aligned}
$$

Adopting the GLR principle yields the following test statistic:

$$
\begin{aligned}
& \ell_{\mathrm{CD}-\mathrm{GLRT}}(\mathbf{U}) \\
&=\ln p_{1}(\mathbf{U})-\ln p_{0}(\mathbf{U}) \\
&=\sum_{m=1}^{M} \sum_{q=0}^{Q-1}\left\{2 \mathfrak { R } \left\{\frac{\beta}{\sigma_{w}^{2}} \mathbf{s}_{B}^{H}\left(\omega_{q}\right) \widehat{\mathbf{H}}^{*}\left(\omega_{q}\right) \mathbf{r}_{m}\left(\omega_{q}\right)\right.\right. \\
&\left.\quad+\frac{1}{\sigma_{v}^{2}} \mathbf{y}_{m}^{T}\left(\omega_{q}\right) \widehat{\mathbf{H}}^{*}\left(\omega_{q}\right) \mathbf{s}_{A}^{*}\left(\omega_{q}\right)\right\} \\
& \quad-\frac{1}{\sigma_{v}^{2}}\left\|\widehat{\mathbf{H}}\left(\omega_{q}\right) \mathbf{s}_{A}\left(\omega_{q}\right)\right\|^{2} \\
&\left.-\frac{\beta^{2}}{\sigma_{w}^{2}}\left\|\widehat{\mathbf{H}}^{T}\left(\omega_{q}\right) \mathbf{s}_{B}\left(\omega_{q}\right)\right\|^{2}\right\} \\
&= \frac{1}{\sigma_{w}^{2} \sigma_{v}^{2}} \sum_{q=0}^{Q-1}\left\{2 \mathfrak{R}\left\{\operatorname{Tr}\left[\widehat{\mathbf{H}}^{*}\left(\omega_{q}\right) \mathbf{J}\left(\omega_{q}\right)\right]\right\}\right. \\
&-\operatorname{Tr}\left[\widehat{\mathbf{H}}^{T}\left(\omega_{q}\right) \widehat{\mathbf{H}}^{*}\left(\omega_{q}\right) \mathbf{C}\left(\omega_{q}\right)\right] \\
&\left.-\operatorname{Tr}\left[\widehat{\mathbf{H}}^{*}\left(\omega_{q}\right) \widehat{\mathbf{H}}^{T}\left(\omega_{q}\right) \mathbf{E}\left(\omega_{q}\right)\right]\right\}
\end{aligned}
$$

\section{Equatin}

Equating to zero yields the Lyapunov Sylvester matrix equation

$$
\widehat{\mathbf{H}}\left(\omega_{q}\right) \mathbf{C}^{T}\left(\omega_{q}\right)+\mathbf{E}^{T}\left(\omega_{q}\right) \hat{\mathbf{H}}\left(\omega_{q}\right)=\mathbf{J}^{T}\left(\omega_{q}\right) .
$$

A straightforward numerical approach to the above equation forms the single vector equation using Kronecker products as follows:

$$
\begin{aligned}
\operatorname{vec}\left\{\mathbf{J}^{T}\left(\omega_{q}\right)\right\} & =\mathbf{L}\left(\omega_{q}\right) \operatorname{vec}\left\{\hat{\mathbf{H}}\left(\omega_{q}\right)\right\} \\
\mathbf{L}\left(\omega_{q}\right) & =\mathbf{C}\left(\omega_{q}\right) \otimes \mathbf{I}_{N}+\mathbf{I}_{P} \otimes \mathbf{E}^{T}\left(\omega_{q}\right)
\end{aligned}
$$

which leads to

$$
\operatorname{vec}\left\{\hat{\mathbf{H}}\left(\omega_{q}\right)\right\}=\left[\mathbf{L}\left(\omega_{q}\right)\right]^{-1} \operatorname{vec}\left\{\mathbf{J}^{T}\left(\omega_{q}\right)\right\} .
$$

The maximum-likelihood estimate of $\hat{\mathbf{H}}\left(\omega_{q}\right)$ for the CD-GLRT can be obtained by un-doing the vectorization operator in (154), see similar comments below (140).

\section{APPENDIX III}

ENERGY NORMALIZATION SCALAR $k_{m}$ IS APPROXIMATELY DETERMINISTIC

The scalar $k_{m}$ in (17) is a random variable because of its dependence on the noise contained in the time-reversed signal. In the development of the TR-GLRT statistic, we assume that $k_{m}$ is a deterministic value. We show here that this is a reasonable assumption. The second order moment of $k_{m}$ is

$$
E\left\{k_{m}^{2}\right\}=E\left\{\frac{\sum_{q=0}^{Q-1}\left\|\mathbf{s}_{A}\left(\omega_{q}\right)\right\|^{2}}{\sum_{q=0}^{Q-1}\left\|\mathbf{H}\left(\omega_{q}\right) \mathbf{s}_{A}\left(\omega_{q}\right)+\mathbf{v}_{m}\left(\omega_{q}\right)\right\|^{2}}\right\}
$$


Applying (103)-(155) yields

$$
E\left\{k_{m}^{2}\right\} \approx \frac{\sum_{q=0}^{Q-1}\left\|\mathbf{s}_{A}\left(\omega_{q}\right)\right\|^{2}}{N Q \sigma_{v}^{2}+\sum_{q=0}^{Q-1}\left\|\mathbf{H}\left(\omega_{q}\right) \mathbf{s}_{A}\left(\omega_{q}\right)\right\|^{2}} .
$$

Assuming that $\mathbf{s}_{A}\left(\omega_{q}\right)=S\left(\omega_{q}\right) \mathbf{1}_{N}$, and $S\left(\omega_{q}\right)=1$, we obtain

$$
E\left\{k_{m}^{2}\right\} \approx \frac{1}{\sigma_{v}^{2}+\frac{1}{N Q} \sum_{q=0}^{Q-1}\left\|\mathbf{H}\left(\omega_{q}\right) \mathbf{1}_{N}\right\|^{2}} .
$$

From the definition of SNR (106), at high SNR, $k_{m} \rightarrow k$, the variance of $k_{m}$ is $\operatorname{Var}\left\{k_{m}\right\} \rightarrow 0$. At low SNR, we use the following approximation. Notice that $k_{m}$ is the inverse of the generalized Rayleigh variate. Since the $p$ th moment of a generalized Rayleigh variate with $f$ degrees of freedom and noncentrality parameter $L$ is [53]

$$
\mu_{p}^{\prime}=2^{\frac{p}{2}} \Gamma\left(\frac{f}{2}+\frac{p}{2}\right) e^{-\frac{L}{2} \frac{1 F_{1}\left(\frac{f}{2}+\frac{p}{2}, \frac{f}{2} ; \frac{L}{2}\right)}{\Gamma\left(\frac{f}{2}\right)}}
$$

where ${ }_{1} F_{1}(\cdot)$ is an hypergeometric function. As an approximation when $f \rightarrow \infty$, and choosing $p=1$, we have the approximate first order moment [53] $\mu_{1}^{\prime} \approx \sqrt{f} e^{\left(L^{2}\right) /(2 f)}$. In our case,

$$
\begin{aligned}
f & =N Q \sigma_{v}^{2}, \\
L^{2} & =\sum_{q=0}^{Q-1}\left\|\mathbf{H}\left(\omega_{q}\right) \mathbf{s}_{A}\left(\omega_{q}\right)\right\|^{2}
\end{aligned}
$$

which yields

$$
\begin{aligned}
\left(E\left\{k_{m}\right\}\right)^{2} & \approx \frac{\sum_{q=0}^{Q-1}\left\|\mathbf{s}_{A}\left(\omega_{q}\right)\right\|^{2}}{\left(\mu_{1}^{\prime}\right)^{2}} \\
& \approx \frac{\sum_{q=0}^{Q-1}\left\|\mathbf{s}_{A}\left(\omega_{q}\right)\right\|^{2}}{N Q \sigma_{v}^{2}} e^{-\frac{\sum_{q=0}^{Q-1}\left\|\mathbf{H}\left(\omega_{q}\right) \mathbf{s}_{A}\left(\omega_{q}\right)\right\|^{2}}{N Q \sigma_{v}^{2}}}
\end{aligned}
$$

Applying the approximation $e^{x} \approx 1+x$, for $|x| \ll 1$, we have

$$
\left(E\left\{k_{m}\right\}\right)^{2} \approx \frac{1}{\sigma_{v}^{2}\left(1+\frac{\sum_{q=0}^{Q-1}\left\|\mathbf{H}\left(\omega_{q}\right) \mathbf{1}_{N}\right\|^{2}}{N Q \sigma_{v}^{2}}\right)} .
$$

Thus, by $\operatorname{Var}\left\{k_{m}\right\}=E\left\{k_{m}^{2}\right\}-\left(E\left\{k_{m}\right\}\right)^{2}$, and using (157) and (163), we conclude that $\operatorname{Var}\left\{k_{m}\right\} \rightarrow 0$ at low SNR. Our analysis shows that at high SNR or low SNR, the variance of $k_{m}$ is small. In Section VII, we see that the receiver operating characteristic (ROC) curves lie in a relatively low SNR range, in which case $k_{m}$ can be safely considered to be deterministic.

\section{REFERENCES}

[1] T. M. Cover and J. A. Thomas, Elements of Information Theory. New York: Wiley, 1991.

[2] J. M. F. Moura and Y. Jin, "Detection by time reversal: Single antenna," IEEE Trans. Signal Process., vol. 55, no. 1, pp. 187-201, Jan. 2007.

[3] M. Fink, "Time reversed acoustics," Phys. Today, vol. 50, no. 3, pp. 34-40, 1997.

[4] N. Chakround, M. Fink, and F. Wu, "Time reversal processing in ultrasonic nondestructive testing," IEEE Trans. Ultrason., Ferroelectr., Freq. Control, vol. 42, no. 6, pp. 1087-1098, Nov. 1995.

[5] C. Dorme and M. Fink, "Ultrasonic beam steering through inhomogeneous layers with a time reversal mirror," IEEE Trans. Ultrason., Ferroelectr., Freq. Control, vol. 43, no. 1, pp. 167-175, Jan. 1996.

[6] H. C. Song, W. A. Kuperman, W. S. Hodgkiss, T. Akal, and C. Ferla, "Iterative time reversal in the ocean," J. Acoustic. Soc. Amer., vol. 105, no. 6, pp. 3176-3184, Jun. 1999.

[7] W. A. Kuperman, W. S. Hodgkiss, and H. C. Song, "Phase conjugation in the ocean: Experimental demonstration of an acoustic time-reversal mirror," J. Acoustic. Soc. Amer., vol. 103, no. 1, pp. 25-40, Jan. 1998

[8] M. Fink, "Time reversal of ultrasonic fields. Part I: Basic principles," IEEE Trans. Ultrason., Ferroelectr., Freq. Control, vol. 39, no. 5, pp. 555-566, Sep. 1992.

[9] A. Derode, A. Tourin, J. de Rosny, M. Tanter, S. Yon, and M. Fink, "Taking advantage of multiple scattering to communicate with timereversal antennas," Phys. Rev. Lett., vol. 90, no. 1, p. 014301, Jan. 2003.

[10] C. Oestges, A. D. Kim, G. Papanicolaou, and A. J. Paulraj, "Characterization of space time focusing in time reversed random fields," IEEE Trans. Antennas Propag., vol. 53, no. 1, pp. 283-293, Jan. 2005.

[11] J. M. F. Moura and Y. Jin, "Time reversal imaging by adaptive interference canceling," IEEE Trans. Signal Process., vol. 56, no. 1, pp. 233-247, Jan. 2008.

[12] J. M. F. Moura, Y. Jin, D. Stancil, J. Zhu, A. Cepni, Y. Jiang, and B. Henty, "Array processing using time reversal: Experiments and performance," in Proc. IEEE Int. Conf. Signal Processing (ICASSP), Toulouse, France, May 2006, vol. IV, pp. 1053-1056.

[13] Y. Jin and J. M. F. Moura, "Asymptotic noise analysis of time reversal detection," in Proc. 40th IEEE Asilomar Conf. Signals, Systems, Computers, Pacific Grove, CA, Oct. 2006, pp. 772-776.

[14] B. E. Henty and D. D. Stancil, "Multipath enabled super-resolution for RF and microwave communication using phase-conjugate arrays," Phys. Rev. Lett., vol. 93, no. 24, p. 243904, Dec. 2004.

[15] J. M. F. Moura, Y. Jin, D. Stancil, J. Zhu, A. Cepni, Y. Jiang, and B. Henty, "Single antenna time reversal adaptive interference cancelation," in Proc. IEEE Int. Conf. Signal Processing (ICASSP), Philadelphia, PA, Mar. 2005, vol. IV, pp. 1121-1124.

[16] F. K. Gruber, E. A. Marengo, and A. J. Devaney, "Time-reversa imaging with multiple signal classification considering multiple scattering between the targets," J. Acoustic. Soc. Amer., vol. 115, no. 6, pp. 3042-3047, Jun. 2004

[17] A. J. Devaney, "Time reversal imaging of obscured targets from multistatic data," IEEE Trans. Antennas Propag., vol. 53, no. 5, pp. 1600-1610, May 2005.

[18] E. A. Marengo and F. K. Gruber, "Subspace-based localization and inverse scattering of multiply scattering point targets," EURASIP J. Appl. Signal Process., vol. 2007, no. 1, pp. 192-192, 2007.

[19] J. G. Proakis, Digital Communications, 3rd ed. New York: McGrawHill, 1995.

[20] E. A. Lee and D. G. Messerschmitt, Digital Communication, 2nd ed. Norwell, MA: Kluwer Academic, 1997.

[21] H. L. VanTrees, Detection, Estimation, and Modulation Theory: Part I. New York: Wiley, 1968.

[22] L. L. Scharf, Statistical Signal Processing: Detection, Estimation, and Time Series Analysis. Reading, MA: Addison-Wesley, 1991.

[23] M. Abramowitz and I. A. Stegun, Handbook of Mathematical Functions With Formulas, Graphs, and Mathematical Tables, 9th ed. New York: Dover, 1964.

[24] M. R. Bell, "Information theory and radar waveform design," IEEE Trans. Inf. Theory, vol. 39, no. 5, pp. 1578-1597, Sep. 1993.

[25] D. A. Garren, A. C. Odom, M. K. Osborn, J. S. Goldstein, S. U. Pillai, and J. R. Guerci, "Full polarization matched illumination for target detection and identification," IEEE Trans. Aerosp. Electron. Syst., vol. 38 , no. 3, pp. 824-837, Jul. 2002.

[26] D. A. Garren, A. C. Odom, M. K. Osborn, J. S. Goldstein, S. U. Pillai, and J. R. Guerci, "Enhanced target detection and identification via optimised radar transmission pulse shape," Proc. Inst. Electr. Eng.-Radar, Sonar Navig., vol. 148, no. 3, pp. 130-138, Jun. 2001.

[27] D. T. Giessing, Target Adaptive Matched Illumination Radar: Principles and Applications. London, U.K.: Pereginus, 1986. 
[28] A. Leshem, O. Naparstek, and A. Nehorai, "Information theoretic adaptive radar waveform design for multiple targets," IEEE J. Sel. Topics Signal Process., vol. 1, no. 1, pp. 42-55, Jun. 2007.

[29] T. Varslot, B. Yacizi, C.-E. Yarman, M. Cheney, and L. Scharf, "Time-reversal waveform precoditioning for clutter rejection," in Proc. IEEE Waveform Diversity Design Conf., Orlando, FL, Jun. 2007, pp. $330-334$.

[30] R. A. Horn and C. R. Johnson, Matrix Analysis. Cambridge, U.K.: Cambridge Univ. Press, 1985.

[31] M. Fink, D. Cassereau, A. Derode, C. Prada, P. Roux, M. Tanter, J. Thomas, and F. Wu, "Time reversal acoustics," Rep. Progress Phys., vol. 63, no. 12, pp. 1933-1995, Dec. 2000.

[32] G. Lerosey, J. de Rosny, A. Tourin, A. Derode, G. Montaldo, and M. Fink, "Time reversal of electromagnetic waves," Phys. Rev. Lett., vol. 92, p. 194301, May 2004.

[33] N. L. Johnson and S. Kotz, Continuous Univariate Distributions, 2nd ed. New York: Wiley, 1970, vol. 1, ch. 28.

[34] D. H. Johnson and D. E. Dudgeon, Array Signal Processing: Concepts and Techniques. Englewood Cliffs, NJ: Prentice-Hall, 1993.

[35] R. A. Monzingo and T. W. Miller, Introduction to Adaptive Arrays. New York: Wiley, 1980.

[36] T. Fujioka, "Asymptotic approximations of the inverse moment of the noncentral chi-squared variable," J. Jpn. Statist. Soc., vol. 31, no. 1, pp. 99-109, 2001.

[37] M. L. Puri and P. K. Sen, Nonparametric Methods in Multivariate Analysis. New York: Wiley, 1971.

[38] J. L. Eaves and E. K. Reedy, Principles of Modern Radar. New York: Van Nostrand Reinhold, 1987.

[39] C. W. Therrien, Discrete Random Signals and Statistical Signal Processing. Englewood Cliffs, NJ: Prentice-Hall, 1992.

[40] M. Soumekh, Synthetic Aperture Radar Signal Processing. New York: Wiley, 1999.

[41] W. G. Carrara, R. S. Goodman, and R. M. Majewski, Spotlight Synthetic Aperture Radar: Signal Processing Algorithms. Boston, MA: Artech House, 1995.

[42] S. R. Deraaf, "SAR imaging via modern 2-D spectral estimation methods," IEEE Trans. Image Process., vol. 7, no. 5, pp. 729-761, May 1998.

[43] Y. Jin and J. M. F. Moura, "TR-SAR: Time reversal target focusing in spotlight SAR," in Proc. IEEE Int. Conf. Signal Processing (ICASSP), Honolulu, HI, Apr. 2007, vol. 2, pp. 957-960.

[44] Y. Jin, J. M. F. Moura, M. Mulford, N. O'Donoughue, and A. Samuel, "Time reversal synthetic aperture radar imaging in multipath," in Proc. 41th IEEE Asilomar Conf. Signals, Systems, Computers, Pacific Grove, CA, Nov. 2007, pp. 1812-1816.

[45] D. Nguyen, G. Benitz, J. Kay, B. Orchard, and R. Whiting, "Superresolution HRR ATR with high definition vector imaging," IEEE Trans. Aerosp. Electron. Syst., vol. 37, no. 4, pp. 1267-1286, Oct. 2001.

[46] G. Benitz, "High definition vector imaging," Lincoln Lab. J., vol. 10, no. 2, pp. 147-170, 1997.

[47] M. Soumekh, "Bistatic synthetic aperture radar inversion with application in dynamic object imaging," IEEE Trans. Signal Process., vol. 39, no. 1, pp. 2044-2055, Sep. 1991.

[48] Y. Ding and D. C. M. Jr., "Fast back projection algorithm for bistatic SAR imaging," in Proc. IEEE Int. Conf. Image Processing, Sep. 2002, vol. II, pp. 449-452.

[49] M. H. Hayes, Statistical Digital Signal Processing and Modeling. New York: Wiley, 1996.

[50] G. Golub and C. V. Loan, Matrix Computations. Baltimore, MD: The Johns Hopkins Univ. Press, 1989.

[51] R. H. Bartels and G. W. Stewart, "A solution of the equation AX + $\mathrm{XB}=\mathrm{C}, "$ Commun. ACM, vol. 15 , no. 9, pp. 820-826, 1972.

[52] G. H. Golub, S. Nash, and C. V. Loan, "A Hessenberg-Schur method for the problem AX $+\mathrm{XB}=\mathrm{C}$," IEEE Trans. Autom. Control, vol. AC-24, no. 6, pp. 909-913, Dec. 1979.

[53] J. H. Park, "Moments of the generalized Rayleigh distribution," Quart. Appl. Math., vol. 19, pp. 45-49, 1961.

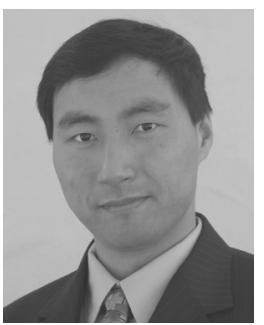

Yuanwei Jin (S'99-M'04-SM'08) received the M.S. and B.S. degrees from East China Normal University, Shanghai, China, in 1993 and 1996, respectively, and the Ph.D. degree in electrical and computer engineering from the University of California at Davis in 2003.

From 2003 to 2004, he was a Visiting Researcher with the University of California at Santa Cruz. From 2004 to 2008, he was a Postdoctoral Research Fellow, then Project Scientist with Department of Electrical and Computer Engineering at Carnegie Mellon University, Pittsburgh, PA. Since August 2008, he has been an Assistant Professor with Department of Engineering and Aviation Sciences at University of Maryland Eastern Shore, Princess Anne, MD. His research interests are in the general area of statistical signal and image processing, with applications in radar/sonar, biomedical imaging, sensor networks, health structure monitoring, and wireless communications. He has published over 30 technical journal and conference papers.

Dr. Jin was a recipient of the Earle C. Anthony Fellowship from the University of California at Davis. He is affiliated with several IEEE societies, Sigma Xi, the American Society for Engineering Education (ASEE), and the American Association for Cancer Research (AACR).

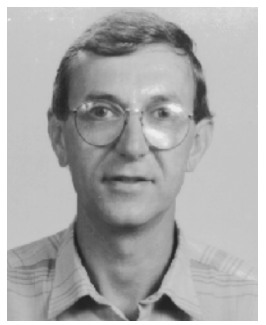

José M. F. Moura (S'71-M'75-SM'90-F'94) received the Engenheiro Electrotécnico degree from the Instituto Superior Técnico (IST), Lisbon, Portugal, and the M.Sc., E.E., and D.Sc. degrees in electrical engineering and computer science from the Massachusetts Institute of Technology (MIT), Cambridge.

$\mathrm{He}$ is a Professor of Electrical and Computer Engineering and, by courtesy, of BioMedical Engineering, at Carnegie Mellon University (CMU), Pittsburgh, PA. He was on the faculty at IST, has held visiting faculty appointments at MIT, and was a visiting research scholar at the University of Southern California. He is a founding codirector of the Center for Sensed Critical Infrastructures Research (CenSCIR) and manages a large education and research program between Carnegie Mellon and Portugal (website: www.icti.cmu.edu). His research interests include statistical and algebraic signal processing, image, bioimaging, and video processing, and digital communications. He has published over 300 technical journal and conference papers, is the co-editor of two books, holds six patents, and has given numerous invited seminars at international conferences, U.S., European, and Japanese universities, and industrial and government Laboratories.

Dr. Moura is the President (2008-2009) of the IEEE Signal Processing Society (SPS) and Vice-Chair of the IEEE Publications Services and Products Board. He was Editor-in-Chief for the IEEE TRANSACTIONS IN Signal PROCESSING, interim Editor-in-Chief for the IEEE SIGNAL Processing LETTERS, and was on the Editorial Board of several journals, including the IEEE Proceedings, the IEEE Signal Processing Magazine, and the ACM Transactions on Sensor Networks. He chaired the IEEE Transactions Committee that joins the Editors-in-Chief of the more than 80 IEEE Transactions and Journals. $\mathrm{He}$ was on the steering and technical committees of numerous conferences. Dr. Moura is a Fellow of the American Association for the Advancement of Science (AAAS), and a corresponding member of the Academy of Sciences of Portugal (Section of Sciences). He was awarded the 2003 IEEE Signal Processing Society Meritorious Service Award and in 2000 the IEEE Millennium Medal. In 2007, he received the CMU's College of Engineering Outstanding Research Award. He is affiliated with several IEEE societies, Sigma Xi, AMS, IMS, and SIAM. 